\title{
Convergence theorems for a system of equilibrium problems and fixed point problems of a strongly nonexpansive sequence
}

\section{Atid Kangtunyakarn*}

"Correspondence:

beawrock@hotmail.com Department of Mathematics, Faculty of Science, King Mongkut's Institute of Technology Ladkrabang, Bangkok 10520, Thailand

\begin{abstract}
The purpose of this paper is to prove a strong convergence theorem of an iterative scheme associated to a strongly nonexpansive sequence for finding a common element of the set of equilibrium problems and the set of fixed point problems of a pair of sequences of nonexpansive mappings where one of them is a strongly nonexpansive sequence. Moreover, in the last section, by using our main result, we obtain a strong convergence theorem of an iterative scheme associated to a strongly nonexpansive sequence for finding a common element of the set of a finite family of equilibrium problems and the set of fixed point problems of a pair of sequences of nonexpansive mappings where one of them is a strongly nonexpansive sequence in a Hilbert space, and we also give some examples to support our main result.
\end{abstract}

Keywords: nonexpansive mappings; strongly nonexpansive sequence; equilibrium problem; fixed point

\section{Introduction}

Throughout this paper, we assume that $H$ is a real Hilbert space with the inner product $\langle\cdot, \cdot\rangle$ and the norm $\|\cdot\|$. A mapping $T$ of $C$ into itself is called nonexpansive if $\|T x-T y\| \leq\|x-y\|$ for all $x, y \in H$. The set of fixed points of $T$ is denoted by $F(T)$, i.e., $F(T)=\{x \in H: T x=$ $x$. It is known that $F(T)$ is closed and convex if $T$ is nonexpansive. Let $P_{C}$ be a metric projection of $H$ onto $C$, i.e., for $x \in H, P_{C} x$ satisfies the property

$$
\left\|x-P_{C} x\right\|=\min _{y \in C}\|x-y\| .
$$

We use " $\rightarrow$ " and " $\rightarrow$ " to denote weak and strong convergence, respectively. Let $\left\{T_{n}\right\}$ be a sequence of mappings of $C$ into $H$. The set of common fixed points of $\left\{T_{n}\right\}$ is denoted by $F\left(\left\{T_{n}\right\}\right)=\bigcap_{n=1}^{\infty} F\left(T_{n}\right)$. Recall the main concepts as follows:

(1) A sequence $\left\{z_{n}\right\}$ in $C$ is said to be an approximate fixed point sequence of $\left\{T_{n}\right\}$ if $z_{n}-T_{n} z_{n} \rightarrow 0$. The set of all bounded approximate fixed point sequences of $\left\{T_{n}\right\}$ is denoted by $\widetilde{F}\left(\left\{T_{n}\right\}\right)$; see [1]. It is clear that if $\left\{T_{n}\right\}$ has a common fixed point, then $\widetilde{F}\left(\left\{T_{n}\right\}\right)$ is nonempty.

\section{Springer}

(c) 2013 Kangtunyakarn; licensee Springer. This is an Open Access article distributed under the terms of the Creative Commons Attribution License (http://creativecommons.org/licenses/by/2.0), which permits unrestricted use, distribution, and reproduction in any medium, provided the original work is properly cited. 
(2) A sequence $\left\{T_{n}\right\}$ is said to be a strongly nonexpansive sequence if each $T_{n}$ is nonexpansive and

$$
x_{n}-y_{n}-\left(T_{n} x_{n}-T_{n} y_{n}\right) \rightarrow 0,
$$

whenever $\left\{x_{n}\right\}$ and $\left\{y_{n}\right\}$ are sequences in $C$ such that $\left\{x_{n}-y_{n}\right\}$ is bounded and $\left\|x_{n}-y_{n}\right\|-\left\|T_{n} x_{n}-T_{n} y_{n}\right\| \rightarrow 0$.

(3) A sequence $\left\{T_{n}\right\}$ having a common fixed point is said to satisfy the condition (Z) if every weak cluster point of $\left\{x_{n}\right\}$ is a common fixed point whenever $\left\{x_{n}\right\} \in \widetilde{F}\left(\left\{T_{n}\right\}\right)$.

(4) A sequence $\left\{T_{n}\right\}$ of nonexpansive mappings of $C$ into $H$ is said to satisfy the condition $(R)$ if

$$
\lim _{n \rightarrow \infty} \sup _{y \in D}\left\|T_{n+1} y-T_{n} y\right\|=0
$$

for every nonempty bounded subset $D$ of $C$; see [2].

Example 1.1 Let $\mathbb{R}$ be a set of real numbers. For every $n \in \mathbb{N}$, the mapping $T_{n}: \mathbb{R} \rightarrow \mathbb{R}$ is defined by $T_{n} x=\frac{1}{n} x$ for all $x \in \mathbb{R}$.

Then $\left\{T_{n}\right\}$ is a nonexpansive sequence, but it is not a strongly nonexpansive sequence.

Example 1.2 For every $n \in \mathbb{N}$, the mapping $T_{n}:[0,1] \rightarrow[0,1]$ is defined by $T_{n} x=\left(1-\frac{1}{n}\right) x$ for all $x \in[0,1]$.

Then $\left\{T_{n}\right\}$ is a strongly nonexpansive sequence.

Solution It is easy to see that $T_{n}$ is a nonexpansive mapping for all $n \in \mathbb{N}$.

Let $\left\{x_{n}\right\}$ and $\left\{y_{n}\right\}$ be sequences in $[0,1]$ with $\left\{x_{n}-y_{n}\right\}$ being bounded and $\left|x_{n}-y_{n}\right|-$ $\left|T_{n} x_{n}-T_{n} y_{n}\right| \rightarrow 0$ as $n \rightarrow \infty$.

Since $x_{n}-y_{n}-\left(T_{n} x_{n}-T_{n} y_{n}\right)=\frac{1}{n}\left(x_{n}-y_{n}\right)$, for all $n \in \mathbb{N}$, then we have

$$
x_{n}-y_{n}-\left(T_{n} x_{n}-T_{n} y_{n}\right) \rightarrow 0 \quad \text { as } n \rightarrow \infty
$$

Then $\left\{T_{n}\right\}$ is a strongly nonexpansive sequence.

Let $G: C \times C \rightarrow \mathbb{R}$ be a bifunction. The equilibrium problem for $G$ is to determine its equilibrium points, i.e., the set

$$
E P(G)=\{x \in G: G(x, y) \geq 0, \forall y \in C\} .
$$

It is a unified model of several problems, namely, variational inequality problem, complementary problem, saddle point problem, optimization problem, fixed point problem, etc.; see [3-5]. Several iterative methods have been proposed to solve the equilibrium problem; see, for instance, [6-8]. In 2005, Combettes and Hirstoaga [4] introduced some iterative schemes of finding the best approximation to the initial data when $E P(G)$ is nonempty and proved a strong convergence theorem.

Also in [4], Combettes and Hiratoaga, following [3], defined $S_{r}: H \rightarrow C$ by

$$
S_{r}(x)=\left\{z \in C: G(z, y)+\frac{1}{r}\langle y-z, z-x\rangle \geq 0, \forall y \in C\right\} .
$$


They proved that under suitable hypotheses $S_{r}$ is single-valued and firmly nonexpansive with $F\left(S_{r}\right)=E P(G)$.

In 2007, Takahashi and Takahashi [9] proved the following theorem.

Theorem 1.3 Let $C$ be a nonempty closed convex subset of $H$. Let $G$ be a bifunction from $C \times C$ to $\mathbb{R}$ satisfying

(A1) $G(x, x)=0, \forall x \in C$;

(A2) $G$ is monotone, i.e., $G(x, y)+G(y, x) \leq 0, \forall x, y \in C$;

(A3) $\forall x, y, z \in C, \lim _{t \rightarrow 0^{+}} G(t z+(1-t) x, y) \leq G(x, y)$;

(A4) $\forall x \in C, y \mapsto G(x, y)$ is convex and lower semicontinuous;

and let $S$ be a nonexpansive mapping of $C$ into $H$ such that $F(S) \cap E P(G) \neq \emptyset$. Let $f$ be a contraction of $H$ into itself, and let $\left\{x_{n}\right\}$ and $\left\{u_{n}\right\}$ be sequences generated by $x_{1} \in H$ and

$$
\begin{aligned}
& G\left(u_{n}, y\right)+\frac{1}{r_{n}}\left\langle y-u_{n}, u_{n}-x_{n}\right\rangle \geq 0, \quad \forall y \in C, \\
& x_{n+1}=\alpha_{n} f\left(x_{n}\right)+\left(1-\alpha_{n}\right) S u_{n}
\end{aligned}
$$

for all $n \in \mathbb{N}$, where $\left\{\alpha_{n}\right\} \subset[0,1]$ and $\left\{r_{n}\right\} \subset(0,1)$ satisfy

(C1) $\lim _{n \rightarrow \infty} \alpha_{n}=0$;

(C2) $\sum_{n=1}^{\infty} \alpha_{n}=\infty$;

(C3) $\sum_{n=1}^{\infty}\left|\alpha_{n+1}-\alpha_{n}\right|<\infty$; and $\liminf _{n \rightarrow \infty} r_{n}>0$ and $\sum_{n=1}^{\infty}\left|r_{n+1}-r_{n}\right|<\infty$.

Then $\left\{x_{n}\right\}$ and $\left\{u_{n}\right\}$ converge strongly to $z \in F(S) \cap E P(G)$, where $z=P_{F(S) \cap E P(G)} f(z)$.

Very recently, in 2011, Aoyama and Kimura [10] proved a strong convergence theorem of the iterative scheme of $\left\{x_{n}\right\}$ associated to a strongly nonexpansive sequence as follows.

Theorem 1.4 Let $H$ be a Hilbert space, let $C$ be a nonempty closed convex subset of $H$, and let $\left\{S_{n}\right\}$ and $\left\{T_{n}\right\}$ be sequences of nonexpansive self-mappings of $C$. Suppose that $F=$ $F\left(\left\{S_{n}\right\}\right) \cap F\left(\left\{T_{n}\right\}\right)$ is nonempty, both $\left\{S_{n}\right\}$ and $\left\{T_{n}\right\}$ satisfy the conditions $(R)$ and $(Z)$, and $\left\{S_{n}\right\}$ or $\left\{T_{n}\right\}$ is a strongly nonexpansive sequence. Let $\left\{\alpha_{n}\right\}$ and $\left\{\beta_{n}\right\}$ be sequences in $[0,1]$ such that

$$
\lim _{n \rightarrow \infty} \alpha_{n}=0, \quad \sum_{n=1}^{\infty} \alpha_{n}=\infty \quad \text { and } \quad 0<\liminf _{n \rightarrow \infty} \beta_{n} \leq \limsup _{n \rightarrow \infty} \beta_{n}<1 .
$$

Let $x, u \in C$ and let $\left\{x_{n}\right\}$ be a sequence in $C$ defined by $x_{1}=x \in C$ and

$$
x_{n+1}=\beta_{n} x_{n}+\left(1-\beta_{n}\right) S_{n}\left(\alpha_{n} u+\left(1-\alpha_{n}\right) T_{n} x_{n}\right)
$$

for all $n \in \mathbb{N}$. Then $\left\{x_{n}\right\}$ converges strongly to $P_{F} u$.

For $x_{1}, u, v \in C$, let $\left\{u_{n}\right\},\left\{v_{n}\right\},\left\{y_{n}\right\}$ and $\left\{x_{n}\right\}$ be the sequences defined by

$$
\left\{\begin{array}{l}
F_{1}\left(u_{n}, u\right)+\frac{1}{r_{n}}\left\langle u-u_{n}, u_{n}-x_{n}\right\rangle \geq 0, \\
F_{2}\left(v_{n}, v\right)+\frac{1}{s_{n}}\left\langle v-v_{n}, v_{n}-x_{n}\right\rangle \geq 0, \\
y_{n}=\delta_{n} u_{n}+\left(1-\delta_{n}\right) v_{n}, \\
x_{n+1}=\beta_{n} x_{n}+\left(1-\beta_{n}\right) S_{n}\left(\alpha_{n} f\left(T_{n} y_{n}\right)+\left(1-\alpha_{n}\right) T_{n} y_{n}\right), \quad \forall n \geq 1,
\end{array}\right.
$$


where $f: C \rightarrow C$ is a contractive mapping with $\alpha \in\left(0, \frac{1}{2}\right)$ and $\left\{S_{n}\right\},\left\{T_{n}\right\}$ are sequences of nonexpansive mappings, one of them is a strongly nonexpansive sequence.

In this paper, inspired and motivated by [10] and [9], we prove that a strong convergence theorem of the iterative scheme $\left\{x_{n}\right\}$ defined by (1.1) converges strongly to $z=P_{\mathbb{F}} f(z)$, where $\mathbb{F}=E P\left(F_{1}\right) \cap E P\left(F_{2}\right) \cap F\left(\left\{S_{n}\right\}\right) \cap F\left(\left\{T_{n}\right\}\right)$, under the conditions $(R)$ and $(Z)$ and suitable conditions of $\left\{r_{n}\right\},\left\{s_{n}\right\},\left\{\alpha_{n}\right\},\left\{\beta_{n}\right\}$ and $\left\{\delta_{n}\right\}$.

\section{Preliminaries}

In this section, we need the following lemmas to prove our main result in the next section.

Lemma 2.1 (See [11]) Given $x \in H$ and $y \in C$. Then $P_{C} x=y$ if and only if the following inequality holds:

$$
\langle x-y, y-z\rangle \geq 0, \quad \forall z \in C
$$

Lemma 2.2 (See [12]) Let $\left\{s_{n}\right\}$ be a sequence of nonnegative real numbers satisfying

$$
s_{n+1}=\left(1-\alpha_{n}\right) s_{n}+\alpha_{n} \beta_{n}, \quad \forall n \geq 0,
$$

where $\left\{\alpha_{n}\right\},\left\{\beta_{n}\right\}$ satisfy the conditions

(1) $\left\{\alpha_{n}\right\} \subset[0,1], \sum_{n=1}^{\infty} \alpha_{n}=\infty$;

(2) $\lim \sup _{n \rightarrow \infty} \beta_{n} \leq 0$ or $\sum_{n=1}^{\infty}\left|\alpha_{n} \beta_{n}\right|<\infty$.

Then $\lim _{n \rightarrow \infty} s_{n}=0$.

Lemma 2.3 (See [13]) Let $\left\{x_{n}\right\}$ and $\left\{z_{n}\right\}$ be bounded sequences in a Banach space $X$, and let $\left\{\beta_{n}\right\}$ be a sequence in $[0,1]$ with $0<\liminf _{n \rightarrow \infty} \beta_{n} \leq \limsup _{n \rightarrow \infty} \beta_{n}<1$. Suppose that

$$
x_{n+1}=\beta_{n} x_{n}+\left(1-\beta_{n}\right) z_{n}
$$

for all integers $n \geq 0$ and

$$
\limsup _{n \rightarrow \infty}\left(\left\|z_{n+1}-z_{n}\right\|-\left\|x_{n+1}-x_{n}\right\|\right) \leq 0
$$

Then $\lim _{n \rightarrow \infty}\left\|x_{n}-z_{n}\right\|=0$.

Lemma 2.4 (See [14]) Let $C$ be a closed convex subset of a strictly convex Banach space $E$. Let $\left\{T_{n}: n \in \mathbb{N}\right\}$ be a sequence of nonexpansive mappings on C. Suppose that $\bigcap_{n=1}^{\infty} F\left(T_{n}\right)$ is nonempty. Let $\left\{\lambda_{n}\right\}$ be a sequence of positive numbers with $\sum_{n=1}^{\infty} \lambda_{n}=1$. Then a mapping $S$ on $C$ defined by

$$
S(x)=\sum_{n=1}^{\infty} \lambda_{n} T_{n} x
$$

for all $x \in C$ is well defined, nonexpansive and $F(S)=\bigcap_{n=1}^{\infty} F\left(T_{n}\right)$ holds.

Lemma 2.5 (See [4]) Let $C$ be a nonempty closed convex subset of a Hilbert space H, and let $G: C \times C \rightarrow \mathbb{R}$ satisfy 
(A1) $G(x, x)=0, \forall x \in C$;

(A2) $G$ is monotone, i.e., $G(x, y)+G(y, x) \leq 0, \forall x, y \in C$;

(A3) $\forall x, y, z \in C, \lim _{t \rightarrow 0^{+}} G(t z+(1-t) x, y) \leq G(x, y)$;

(A4) $\forall x \in C, y \mapsto G(x, y)$ is convex and lower semicontinuous.

For $x \in H$ and $r>0$, define a mapping $S_{r}: H \rightarrow C$ as follows:

$$
S_{r}(x)=\left\{z \in C: G(z, y)+\frac{1}{r}\langle y-z, z-x\rangle \geq 0, \forall y \in C\right\} .
$$

Then $S_{r}$ is well defined and the following hold:

(1) $S_{r}$ is single-valued;

(2) $S_{r}$ is firmly nonexpansive, i.e., $\left\|S_{r}(x)-S_{r}(y)\right\|^{2} \leq\left\langle S_{r}(x)-S_{r}(y), x-y\right\rangle, \forall x, y \in H$;

(3) $F\left(S_{r}\right)=E P(G)$

(4) $E P(G)$ is closed and convex.

Lemma 2.6 (See [11]) (Demiclosedness principle) Assume that $T$ is a nonexpansive selfmapping of a closed convex subset $C$ of a Hilbert space $H$. If $T$ has a fixed point, then $I-T$ is demiclosed. That is, whenever $\left\{x_{n}\right\}$ is a sequence in $C$ weakly converging to some $x \in C$ and the sequence $\left\{(I-T) x_{n}\right\}$ converges strongly to some $y$, it follows that $(I-T) x=y$. Here, $I$ is the identity mapping of $H$.

Lemma 2.7 Let $H$ be a real Hilbert space. Then, for all $x, y \in H$,

$$
\|x+y\|^{2} \leq\|x\|^{2}+2\langle y, x+y\rangle .
$$

Lemma 2.8 (See [10]) Let $H$ be a Hilbert space, let $C$ be a nonempty subset of $H$, and let $\left\{S_{n}\right\}$ and $\left\{T_{n}\right\}$ be the sequences of nonexpansive self-mappings of $C$. Suppose that $\left\{S_{n}\right\}$ and $\left\{T_{n}\right\}$ satisfy the condition $(R)$ and that $\left\{T_{n} y: n \in \mathbb{N}, y \in D\right\}$ is bounded for any bounded subset $D$ of $C$. Then $\left\{S_{n} T_{n}\right\}$ satisfies the condition $(R)$.

Lemma 2.9 (See [1]) Let $H$ be a Hilbert space, let $C$ be a nonempty subset of $H$, and let $\left\{S_{n}\right\}$ and $\left\{T_{n}\right\}$ be the sequences of nonexpansive self-mappings of $C$. Suppose that $\left\{S_{n}\right\}$ or $\left\{T_{n}\right\}$ is a strongly nonexpansive sequence and that $\widetilde{F}\left(\left\{S_{n}\right\}\right) \cap \widetilde{F}\left(\left\{T_{n}\right\}\right)$ is nonempty. Then $\widetilde{F}\left(\left\{S_{n}\right\}\right) \cap \widetilde{F}\left(\left\{T_{n}\right\}\right)=\widetilde{F}\left(\left\{S_{n} T_{n}\right\}\right)$.

\section{Main result}

Theorem 3.1 Let $H$ be a Hilbert space, let $C$ be a nonempty closed convex subset of $H$. Let $F_{1}$ and $F_{2}$ be two bifunctions from $C \times C$ into $\mathbb{R}$ satisfying (A1)-(A4), respectively, and let $\left\{S_{n}\right\}$ and $\left\{T_{n}\right\}$ be sequences of nonexpansive self-mappings of $C$ with $\mathbb{F}=E P\left(F_{1}\right) \cap E P\left(F_{2}\right) \cap$ $F\left(\left\{S_{n}\right\}\right) \cap F\left(\left\{T_{n}\right\}\right) \neq \emptyset$. Let $\left\{T_{n}\right\}$ or $\left\{S_{n}\right\}$ be a sequence of strongly nonexpansive mappings, and let $f: C \rightarrow C$ be a contractive mapping with $\alpha \in\left(0, \frac{1}{2}\right)$. Let $\left\{x_{n}\right\},\left\{u_{n}\right\},\left\{v_{n}\right\}$ be sequences generated by $x_{1}, u, v \in C$ and

$$
\left\{\begin{array}{l}
F_{1}\left(u_{n}, u\right)+\frac{1}{r_{n}}\left\langle u-u_{n}, u_{n}-x_{n}\right\rangle \geq 0, \\
F_{2}\left(v_{n}, v\right)+\frac{1}{s_{n}}\left\langle v-v_{n}, v_{n}-x_{n}\right\rangle \geq 0, \\
y_{n}=\delta_{n} u_{n}+\left(1-\delta_{n}\right) v_{n}, \\
x_{n+1}=\beta_{n} x_{n}+\left(1-\beta_{n}\right) S_{n}\left(\alpha_{n} f\left(T_{n} y_{n}\right)+\left(1-\alpha_{n}\right) T_{n} y_{n}\right), \quad \forall n \geq 1,
\end{array}\right.
$$


where $\left\{\alpha_{n}\right\},\left\{\beta_{n}\right\} \in[0,1],\left\{r_{n}\right\},\left\{s_{n}\right\} \in(a, b) \in[0,1]$. Assume that the following conditions hold:

(i) $\lim _{n \rightarrow \infty} \alpha_{n}=0$ and $\sum_{n=1}^{\infty} \alpha_{n}=\infty$;

(ii) $0<\liminf _{n \rightarrow \infty} \beta_{n} \leq \lim \sup _{n \rightarrow \infty} \beta_{n}<1$;

(iii) $\sum_{n=0}^{\infty}\left|r_{n+1}-r_{n}\right|, \sum_{n=0}^{\infty}\left|s_{n+1}-s_{n}\right|<\infty$;

(iv) $\lim _{n \rightarrow \infty} \delta_{n}=\delta \in(0,1)$;

(v) $\left\{S_{n}\right\}$ and $\left\{T_{n}\right\}$ satisfy the conditions $R$ and $Z$.

Then the sequences $\left\{x_{n}\right\},\left\{u_{n}\right\},\left\{v_{n}\right\},\left\{y_{n}\right\}$ converge strongly to $z=P_{\mathbb{F}} f(z)$.

Proof Let $v \in \mathbb{F}$. From the definition of $x_{n}$, we have

$$
\begin{aligned}
\left\|x_{n+1}-v\right\|= & \left\|\beta_{n}\left(x_{n}-v\right)+\left(1-\beta_{n}\right)\left(S_{n}\left(\alpha_{n} f\left(T_{n} y_{n}\right)+\left(1-\alpha_{n}\right) T_{n} y_{n}\right)-v\right)\right\| \\
\leq & \beta_{n}\left\|x_{n}-v\right\|+\left(1-\beta_{n}\right)\left\|\alpha_{n} f\left(T_{n} y_{n}\right)+\left(1-\alpha_{n}\right) T_{n} y_{n}-v\right\| \\
\leq & \beta_{n}\left\|x_{n}-v\right\|+\left(1-\beta_{n}\right)\left(\alpha_{n}\left\|f\left(T_{n} y_{n}\right)-v\right\|+\left(1-\alpha_{n}\right)\left\|T_{n} y_{n}-v\right\|\right) \\
\leq & \beta_{n}\left\|x_{n}-v\right\|+\left(1-\beta_{n}\right)\left(\alpha_{n}\left\|f\left(T_{n} y_{n}\right)-f(v)\right\|+\alpha_{n}\|f(v)-v\|\right. \\
& \left.+\left(1-\alpha_{n}\right)\left\|T_{n} y_{n}-v\right\|\right) \\
\leq & \beta_{n}\left\|x_{n}-v\right\|+\left(1-\beta_{n}\right)\left(\alpha_{n} \alpha\left\|y_{n}-v\right\|+\alpha_{n}\|f(v)-v\|\right. \\
& \left.+\left(1-\alpha_{n}\right)\left\|y_{n}-v\right\|\right) \\
= & \beta_{n}\left\|x_{n}-v\right\|+\left(1-\beta_{n}\right)\left(\alpha_{n}\|f(v)-v\|\right. \\
& \left.+\left(1-\alpha_{n}(1-\alpha)\right)\left\|y_{n}-v\right\|\right) .
\end{aligned}
$$

From Lemma 2.5 and (3.1), we have $E P\left(F_{1}\right)=F\left(S_{r_{n}}\right), E P\left(F_{2}\right)=F\left(S_{S_{n}}\right), S_{r_{n}} x_{n}=u_{n}$ and $S_{S_{n}} x_{n}=v_{n}$. By $v \in \mathbb{F}$ and the nonexpansiveness of $S_{r_{n}}$ and $S_{s_{n}}$, we have

$$
\begin{aligned}
\left\|y_{n}-v\right\| & =\left\|\delta_{n}\left(u_{n}-v\right)+\left(1-\delta_{n}\right)\left(v_{n}-v\right)\right\| \\
& \leq \delta_{n}\left\|u_{n}-v\right\|+\left(1-\delta_{n}\right)\left\|v_{n}-v\right\| \\
& =\delta_{n}\left\|S_{r_{n}} x_{n}-v\right\|+\left(1-\delta_{n}\right)\left\|S_{S_{n}} x_{n}-v\right\| \\
& \leq\left\|x_{n}-v\right\| .
\end{aligned}
$$

Substituting (3.3) into (3.2), we have

$$
\begin{aligned}
\left\|x_{n+1}-v\right\| \leq & \beta_{n}\left\|x_{n}-v\right\|+\left(1-\beta_{n}\right)\left(\alpha_{n}\|f(v)-v\|\right. \\
& \left.+\left(1-\alpha_{n}(1-\alpha)\right)\left\|y_{n}-v\right\|\right) \\
\leq & \beta_{n}\left\|x_{n}-v\right\|+\left(1-\beta_{n}\right)\left(\alpha_{n}\|f(v)-v\|\right. \\
& \left.+\left(1-\alpha_{n}(1-\alpha)\right)\left\|x_{n}-v\right\|\right) \\
= & \beta_{n}\left\|x_{n}-v\right\|+\left(1-\beta_{n}\right) \alpha_{n}\|f(v)-v\| \\
& +\left(1-\beta_{n}\right)\left(1-\alpha_{n}(1-\alpha)\right)\left\|x_{n}-v\right\| \\
= & \left(1-\beta_{n}\right) \alpha_{n}\|f(v)-v\|+\left(1-\alpha_{n}\left(1-\beta_{n}\right)(1-\alpha)\right)\left\|x_{n}-v\right\| \\
\leq & \max \left\{\left\|x_{n}-v\right\|, \frac{\|f(v)-v\|}{1-\alpha}\right\} .
\end{aligned}
$$


By induction we can conclude that $\left\{x_{n}\right\}$ is bounded and so are $\left\{u_{n}\right\},\left\{v_{n}\right\},\left\{y_{n}\right\}$. Next, we show that $\widetilde{F}\left(\left\{S_{n} A_{n}\right\}\right)=\widetilde{F}\left(\left\{S_{n}\right\}\right)$ and $\widetilde{F}\left(\left\{A_{n} T_{n}\right\}\right)=\widetilde{F}\left(\left\{T_{n}\right\}\right)$, where $A_{n}=\alpha_{n} f+\left(1-\alpha_{n}\right) I$.

Let $\left\{z_{n}\right\}$ be a bounded sequence in $C$. From the nonexpansiveness of $S_{n}$, we have

$$
\left\|S_{n} A_{n} z_{n}-S_{n} z_{n}\right\| \leq\left\|A_{n} z_{n}-z_{n}\right\|=\alpha_{n}\left\|f\left(z_{n}\right)-z_{n}\right\| .
$$

From (3.4) and $\alpha_{n} \rightarrow 0$ as $n \rightarrow \infty$, we have

$$
\lim _{n \rightarrow \infty}\left\|S_{n} A_{n} z_{n}-S_{n} z_{n}\right\|=0
$$

Let $\left\{z_{n}\right\} \in \widetilde{F}\left(\left\{S_{n} A_{n}\right\}\right)$, then we have

$$
\left\|z_{n}-S_{n} z_{n}\right\| \leq\left\|z_{n}-S_{n} A_{n} z_{n}\right\|+\left\|S_{n} A_{n} z_{n}-S_{n} z_{n}\right\| .
$$

From (3.5), we have

$$
\lim _{n \rightarrow \infty}\left\|z_{n}-S_{n} z_{n}\right\|=0
$$

which implies that $\left\{z_{n}\right\} \in \widetilde{F}\left(\left\{S_{n}\right\}\right)$. It follows that

$$
\widetilde{F}\left(\left\{S_{n} A_{n}\right\}\right) \subseteq \widetilde{F}\left(\left\{S_{n}\right\}\right)
$$

Let $\left\{z_{n}\right\} \in \widetilde{F}\left(\left\{S_{n}\right\}\right)$, then we have

$$
\left\|z_{n}-S_{n} A_{n} z_{n}\right\| \leq\left\|z_{n}-S_{n} z_{n}\right\|+\left\|S_{n} z_{n}-S_{n} A_{n} z_{n}\right\| .
$$

From (3.5), we have

$$
\lim _{n \rightarrow \infty}\left\|z_{n}-S_{n} A_{n} z_{n}\right\|=0,
$$

which implies that $\left\{z_{n}\right\} \in \widetilde{F}\left(\left\{S_{n} A_{n}\right\}\right)$. It follows that

$$
\widetilde{F}\left(\left\{S_{n}\right\}\right) \subseteq \widetilde{F}\left(\left\{S_{n} A_{n}\right\}\right) .
$$

From (3.6) and (3.7), we have

$$
\widetilde{F}\left(\left\{S_{n}\right\}\right)=\widetilde{F}\left(\left\{S_{n} A_{n}\right\}\right) .
$$

Let $\left\{z_{n}\right\}$ be a bounded sequence in $C$, then we have $\left\{T_{n} z_{n}\right\}$ is bounded and so is $\left\{f\left(T_{n} z_{n}\right)\right\}$. Since

$$
\left\|A_{n} T_{n} z_{n}-T_{n} z_{n}\right\|=\alpha_{n}\left\|f\left(T_{n} z_{n}\right)-T_{n} z_{n}\right\|
$$

and $\alpha_{n} \rightarrow 0$ as $n \rightarrow \infty$, we have

$$
\lim _{n \rightarrow \infty}\left\|A_{n} T_{n} z_{n}-T_{n} z_{n}\right\|=0 .
$$


Let $\left\{z_{n}\right\} \in \widetilde{F}\left(\left\{A_{n} T_{n}\right\}\right)$, then we have

$$
\left\|z_{n}-T_{n} z_{n}\right\| \leq\left\|z_{n}-A_{n} T_{n} z_{n}\right\|+\left\|A_{n} T_{n} z_{n}-T_{n} z_{n}\right\| .
$$

From (3.9), we have

$$
\lim _{n \rightarrow \infty}\left\|z_{n}-T_{n} z_{n}\right\|=0
$$

which implies that

$$
\left\{z_{n}\right\} \in \widetilde{F}\left(\left\{T_{n}\right\}\right)
$$

It follows that

$$
\widetilde{F}\left(\left\{A_{n} T_{n}\right\}\right) \subseteq \widetilde{F}\left(\left\{T_{n}\right\}\right) .
$$

Let $\left\{z_{n}\right\} \in \widetilde{F}\left(\left\{T_{n}\right\}\right)$, then we have

$$
\left\|z_{n}-A_{n} T_{n} z_{n}\right\| \leq\left\|z_{n}-T_{n} z_{n}\right\|+\left\|T_{n} z_{n}-A_{n} T_{n} z_{n}\right\| .
$$

From (3.9), we have

$$
\lim _{n \rightarrow \infty}\left\|z_{n}-A_{n} T_{n} z_{n}\right\|=0
$$

which implies that

$$
\left\{z_{n}\right\} \in \widetilde{F}\left(\left\{A_{n} T_{n}\right\}\right) .
$$

It follows that

$$
\widetilde{F}\left(\left\{T_{n}\right\}\right) \subseteq \widetilde{F}\left(\left\{A_{n} T_{n}\right\}\right) .
$$

From (3.10) and (3.11), we have

$$
\widetilde{F}\left(\left\{T_{n}\right\}\right)=\widetilde{F}\left(\left\{A_{n} T_{n}\right\}\right) .
$$

Next, we show that

$$
\widetilde{F}\left(\left\{S_{n} A_{n} T_{n}\right\}\right)=\widetilde{F}\left(\left\{S_{n}\right\}\right) \cap \widetilde{F}\left(\left\{T_{n}\right\}\right) .
$$

Since $\mathbb{F}$ is nonempty, from (3.8), (3.12), we have

$$
\widetilde{F}\left(\left\{S_{n} A_{n}\right\}\right) \cap \widetilde{F}\left(\left\{T_{n}\right\}\right)=\widetilde{F}\left(\left\{S_{n}\right\}\right) \cap \widetilde{F}\left(\left\{T_{n}\right\}\right) \neq \emptyset
$$

and

$$
\widetilde{F}\left(\left\{S_{n}\right\}\right) \cap \widetilde{F}\left(\left\{A_{n} T_{n}\right\}\right)=\widetilde{F}\left(\left\{S_{n}\right\}\right) \cap \widetilde{F}\left(\left\{T_{n}\right\}\right) \neq \emptyset .
$$


Suppose that $\left\{S_{n}\right\}$ is a strongly nonexpansive sequence. From (3.14) and Lemma 2.9, we have

$$
\widetilde{F}\left(\left\{S_{n} A_{n} T_{n}\right\}\right)=\widetilde{F}\left(\left\{S_{n}\right\}\right) \cap \widetilde{F}\left(\left\{A_{n} T_{n}\right\}\right)=\widetilde{F}\left(\left\{S_{n}\right\}\right) \cap \widetilde{F}\left(\left\{T_{n}\right\}\right) .
$$

On the other hand, suppose that $\left\{T_{n}\right\}$ is a strongly nonexpansive sequence. From (3.13) and Lemma 2.9, we have

$$
\widetilde{F}\left(\left\{S_{n} A_{n} T_{n}\right\}\right)=\widetilde{F}\left(\left\{S_{n} A_{n}\right\}\right) \cap \widetilde{F}\left(\left\{T_{n}\right\}\right)=\widetilde{F}\left(\left\{S_{n}\right\}\right) \cap \widetilde{F}\left(\left\{T_{n}\right\}\right) .
$$

From (3.16) and (3.15), we have $\widetilde{F}\left(\left\{S_{n} A_{n} T_{n}\right\}\right)=\widetilde{F}\left(\left\{S_{n}\right\}\right) \cap \widetilde{F}\left(\left\{T_{n}\right\}\right)$. Next, we show that $\left\{A_{n}\right\}$ and $\left\{S_{n} A_{n} T_{n}\right\}$ satisfy the condition $(R)$. It is easy to see that $A_{n}$ is a nonexpansive mapping for every $n \in \mathbb{N}$ and that $\left\{A_{n} y: n \in \mathbb{N}, y \in D\right\}$ is bounded, where $D$ is a bounded subset of $C$. Let $y \in D$, then we have

$$
\begin{aligned}
\left\|A_{n+1} y-A_{n} y\right\| & =\left\|\alpha_{n+1} f(y)+\left(1-\alpha_{n+1}\right) y-\alpha_{n} f(y)-\left(1-\alpha_{n}\right) y\right\| \\
& \leq\left|\alpha_{n+1}-\alpha_{n}\right|\|f(y)\|+\left|\alpha_{n+1}-\alpha_{n}\right|\|y\| .
\end{aligned}
$$

From the condition (i), we have

$$
\lim _{n \rightarrow \infty} \sup _{y \in D}\left\|A_{n+1} y-A_{n} y\right\|=0 .
$$

It follows that $\left\{A_{n}\right\}$ satisfies the condition $(R)$. From Lemma 2.8, we have that $\left\{S_{n} A_{n}\right\}$ satisfies the condition $(R)$. From the nonexpansiveness of $T_{n}$ and $\mathbb{F} \neq \emptyset$, we have $\left\{T_{n} y: n \in \mathbb{N}, y \in\right.$ $D$ \} is bounded for any bounded subset $D$ of $C$. From Lemma 2.8, we have that $\left\{S_{n} A_{n} T_{n}\right\}$ satisfies the condition $(R)$.

Next, we show that

$$
\lim _{n \rightarrow \infty}\left\|x_{n+1}-x_{n}\right\|=0
$$

Put

$$
x_{n+1}=\beta_{n} x_{n}+\left(1-\beta_{n}\right) w_{n},
$$

where $w_{n}=S_{n}\left(\alpha_{n} f\left(T_{n} y_{n}\right)+\left(1-\alpha_{n}\right) T_{n} y_{n}\right)$. From the definition of $w_{n}$, we have

$$
\begin{aligned}
\left\|w_{n+1}-w_{n}\right\| & =\left\|S_{n+1} A_{n+1} T_{n+1} y_{n+1}-S_{n} A_{n} T_{n} y_{n}\right\| \\
& \leq\left\|S_{n+1} A_{n+1} T_{n+1} y_{n+1}-S_{n} A_{n} T_{n} y_{n+1}\right\|+\left\|S_{n} A_{n} T_{n} y_{n+1}-S_{n} A_{n} T_{n} y_{n}\right\| \\
& \leq \sup _{y \in D}\left\|S_{n+1} A_{n+1} T_{n+1} y-S_{n} A_{n} T_{n} y\right\|+\left\|y_{n+1}-y_{n}\right\|,
\end{aligned}
$$

where $D$ is a bounded subset of $C$. Besides, we have

$$
\begin{aligned}
\left\|y_{n+1}-y_{n}\right\| & =\left\|\delta_{n+1} u_{n+1}+\left(1-\delta_{n+1}\right) v_{n+1}-\delta_{n} u_{n}-\left(1-\delta_{n}\right) v_{n}\right\| \\
& =\| \delta_{n+1} u_{n+1}-\delta_{n+1} u_{n}+\delta_{n+1} u_{n}-\left(1-\delta_{n+1}\right) v_{n}+\left(1-\delta_{n+1}\right) v_{n}
\end{aligned}
$$




$$
\begin{aligned}
& +\left(1-\delta_{n+1}\right) v_{n+1}-\delta_{n} u_{n}-\left(1-\delta_{n}\right) v_{n} \| \\
= & \left\|\delta_{n+1}\left(u_{n+1}-u_{n}\right)+\left(\delta_{n+1}-\delta_{n}\right) u_{n}+\left(1-\delta_{n+1}\right)\left(v_{n+1}-v_{n}\right)+\left(\delta_{n}-\delta_{n+1}\right) v_{n}\right\| \\
\leq & \delta_{n+1}\left\|u_{n+1}-u_{n}\right\|+\left|\delta_{n+1}-\delta_{n}\right|\left\|u_{n}\right\|+\left(1-\delta_{n+1}\right)\left\|v_{n+1}-v_{n}\right\| \\
& +\left|\delta_{n}-\delta_{n+1}\right|\left\|v_{n}\right\| .
\end{aligned}
$$

From (3.1) and Lemma 2.5, we have $u_{n}=S_{r_{n}} x_{n}$. This implies that

$$
F_{1}\left(u_{n}, u\right)+\frac{1}{r_{n}}\left\langle u-u_{n}, u_{n}-x_{n}\right\rangle \geq 0 \quad \text { for all } u \in C
$$

and

$$
F_{1}\left(u_{n+1}, u\right)+\frac{1}{r_{n+1}}\left\langle u-u_{n+1}, u_{n+1}-x_{n+1}\right\rangle \geq 0 \quad \text { for all } u \in C .
$$

Putting $u=u_{n+1}$ in (3.21) and $u=u_{n}$ in (3.22), we have

$$
F_{1}\left(u_{n}, u_{n+1}\right)+\frac{1}{r_{n}}\left\langle u_{n+1}-u_{n}, u_{n}-x_{n}\right\rangle \geq 0
$$

and

$$
F_{1}\left(u_{n+1}, u_{n}\right)+\frac{1}{r_{n+1}}\left\langle u_{n}-u_{n+1}, u_{n+1}-x_{n+1}\right\rangle \geq 0 .
$$

Summing up the last two inequalities and using (A2), we obtain

$$
\left\langle u_{n+1}-u_{n}, \frac{u_{n}-x_{n}}{r_{n}}-\frac{u_{n+1}-x_{n+1}}{r_{n+1}}\right\rangle \geq 0 .
$$

This implies that

$$
\left\langle u_{n+1}-u_{n}, u_{n}-u_{n+1}+u_{n+1}-x_{n}-\frac{r_{n}}{r_{n+1}}\left(u_{n+1}-x_{n+1}\right)\right\rangle \geq 0 .
$$

Hence,

$$
\begin{aligned}
\left\|u_{n+1}-u_{n}\right\|^{2} & \leq\left\langle u_{n+1}-u_{n}, u_{n+1}-x_{n}-\frac{r_{n}}{r_{n+1}}\left(u_{n+1}-x_{n+1}\right)\right\rangle \\
& =\left\langle u_{n+1}-u_{n}, u_{n+1}-x_{n+1}+x_{n+1}-x_{n}-\frac{r_{n}}{r_{n+1}}\left(u_{n+1}-x_{n+1}\right)\right\rangle \\
& =\left\langle u_{n+1}-u_{n}, x_{n+1}-x_{n}+\left(1-\frac{r_{n}}{r_{n+1}}\right)\left(u_{n+1}-x_{n+1}\right)\right\rangle \\
& \leq\left\|u_{n+1}-u_{n}\right\|\left(\left\|x_{n+1}-x_{n}\right\|+\frac{1}{r_{n+1}}\left|r_{n+1}-r_{n}\right|\left\|u_{n+1}-x_{n+1}\right\|\right) \\
& \leq\left\|u_{n+1}-u_{n}\right\|\left(\left\|x_{n+1}-x_{n}\right\|+\frac{1}{a}\left|r_{n+1}-r_{n}\right|\left\|u_{n+1}-x_{n+1}\right\|\right) .
\end{aligned}
$$

Then we have

$$
\left\|u_{n+1}-u_{n}\right\| \leq\left\|x_{n+1}-x_{n}\right\|+\frac{1}{a}\left|r_{n+1}-r_{n}\right|\left\|u_{n+1}-x_{n+1}\right\| .
$$


From (3.1) and Lemma 2.5, we have $v_{n}=S_{S_{n}} x_{n}$. This implies that

$$
F_{2}\left(v_{n}, v\right)+\frac{1}{s_{n}}\left\langle v-v_{n}, v_{n}-x_{n}\right\rangle \geq 0 \quad \text { for all } v \in C .
$$

By using the same method as (3.25), we have

$$
\left\|v_{n+1}-v_{n}\right\| \leq\left\|x_{n+1}-x_{n}\right\|+\frac{1}{a}\left|s_{n+1}-s_{n}\right|\left\|v_{n+1}-x_{n+1}\right\|
$$

Substituting (3.25) and (3.26) into (3.20), we have

$$
\begin{aligned}
\left\|y_{n+1}-y_{n}\right\| \leq & \delta_{n+1}\left\|u_{n+1}-u_{n}\right\|+\left|\delta_{n+1}-\delta_{n}\right|\left\|u_{n}\right\|+\left(1-\delta_{n+1}\right)\left\|v_{n+1}-v_{n}\right\| \\
& +\left|\delta_{n}-\delta_{n+1}\right|\left\|v_{n}\right\| \\
\leq & \delta_{n+1}\left(\left\|x_{n+1}-x_{n}\right\|+\frac{1}{a}\left|r_{n+1}-r_{n}\right|\left\|u_{n+1}-x_{n+1}\right\|\right) \\
& +\left(1-\delta_{n+1}\right)\left(\left\|x_{n+1}-x_{n}\right\|+\frac{1}{a}\left|s_{n+1}-s_{n}\right|\left\|v_{n+1}-x_{n+1}\right\|\right) \\
& +2 M\left|\delta_{n}-\delta_{n+1}\right| \\
\leq & \left\|x_{n+1}-x_{n}\right\|+\frac{1}{a}\left|r_{n+1}-r_{n}\right|\left\|u_{n+1}-x_{n+1}\right\| \\
& +\frac{1}{a}\left|s_{n+1}-s_{n}\right|\left\|v_{n+1}-x_{n+1}\right\|+2 M\left|\delta_{n}-\delta_{n+1}\right|,
\end{aligned}
$$

where $M=\sup _{n \in \mathbb{N}}\left\{\left\|u_{n}\right\|,\left\|v_{n}\right\|\right\}$. Substituting (3.27) into (3.19), we have

$$
\begin{aligned}
&\left\|w_{n+1}-w_{n}\right\| \leq \sup _{y \in D}\left\|S_{n+1} A_{n+1} T_{n+1} y-S_{n} A_{n} T_{n} y\right\|+\left\|y_{n+1}-y_{n}\right\| \\
& \leq \sup _{y \in D}\left\|S_{n+1} A_{n+1} T_{n+1} y-S_{n} A_{n} T_{n} y\right\|+\left\|x_{n+1}-x_{n}\right\| \\
&+\frac{1}{a}\left|r_{n+1}-r_{n}\right|\left\|u_{n+1}-x_{n+1}\right\| \\
&+\frac{1}{a}\left|s_{n+1}-s_{n}\right|\left\|v_{n+1}-x_{n+1}\right\|+2 M\left|\delta_{n}-\delta_{n+1}\right| .
\end{aligned}
$$

From (3.28), the conditions (iii), (iv) and $\left\{S_{n} A_{n} T_{n}\right\}$ satisfying the condition ( $R$ ), we have

$$
\limsup _{n \rightarrow \infty}\left(\left\|w_{n+1}-w_{n}\right\|-\left\|x_{n+1}-x_{n}\right\|\right) \leq 0
$$

From Lemma 2.3 and the definition of $x_{n}$, we have

$$
\lim _{n \rightarrow \infty}\left\|x_{n}-w_{n}\right\|=0
$$

From the definition of $x_{n}$, we have

$$
x_{n+1}-x_{n}=\left(1-\beta_{n}\right)\left(w_{n}-x_{n}\right) \text {. }
$$

From (3.30), (3.31) and the condition (ii), we have

$$
\lim _{n \rightarrow \infty}\left\|x_{n+1}-x_{n}\right\|=0 .
$$


Next, we show that

$$
\lim _{n \rightarrow \infty}\left\|y_{n}-x_{n}\right\|=0
$$

From the definition of $y_{n}$, we have

$$
\left\|y_{n}-x_{n}\right\| \leq \delta_{n}\left\|u_{n}-x_{n}\right\|+\left(1-\delta_{n}\right)\left\|v_{n}-x_{n}\right\| .
$$

Next, we show that

$$
\lim _{n \rightarrow \infty}\left\|u_{n}-x_{n}\right\|=\lim _{n \rightarrow \infty}\left\|v_{n}-x_{n}\right\|=0 .
$$

Let $v \in \mathbb{F}$. From the definition of $x_{n}$, we have

$$
\begin{aligned}
\left\|x_{n+1}-v\right\|^{2} \leq & \beta_{n}\left\|x_{n}-v\right\|^{2}+\left(1-\beta_{n}\right)\left\|S_{n}\left(\alpha_{n} f\left(T_{n} y_{n}\right)+\left(1-\alpha_{n}\right) T_{n} y_{n}\right)-v\right\|^{2} \\
\leq & \beta_{n}\left\|x_{n}-v\right\|^{2}+\left(1-\beta_{n}\right)\left\|\alpha_{n}\left(f\left(T_{n} y_{n}\right)-v\right)+\left(1-\alpha_{n}\right)\left(T_{n} y_{n}-v\right)\right\|^{2} \\
\leq & \beta_{n}\left\|x_{n}-v\right\|^{2}+\left(1-\beta_{n}\right)\left(\alpha_{n}\left\|f\left(T_{n} y_{n}\right)-v\right\|^{2}+\left(1-\alpha_{n}\right)\left\|T_{n} y_{n}-v\right\|^{2}\right) \\
\leq & \beta_{n}\left\|x_{n}-v\right\|^{2}+\left(1-\beta_{n}\right)\left(\alpha_{n}\left\|f\left(T_{n} y_{n}\right)-v\right\|^{2}+\left(1-\alpha_{n}\right)\left\|y_{n}-v\right\|^{2}\right) \\
\leq & \beta_{n}\left\|x_{n}-v\right\|^{2}+\left(1-\beta_{n}\right)\left(\alpha_{n}\left\|f\left(T_{n} y_{n}\right)-v\right\|^{2}\right. \\
& \left.+\left(1-\alpha_{n}\right)\left(\delta_{n}\left\|u_{n}-v\right\|^{2}+\left(1-\delta_{n}\right)\left\|v_{n}-v\right\|^{2}\right)\right) .
\end{aligned}
$$

From the firm nonexpansiveness of $S_{r_{n}}$ and $u_{n}=S_{r_{n}} x_{n}$, we have

$$
\begin{aligned}
\left\|u_{n}-v\right\|^{2} & =\left\|S_{r_{n}} x_{n}-S_{r_{n}} v\right\|^{2} \\
& \leq\left\langle u_{n}-v, x_{n}-v\right\rangle \\
& =\frac{1}{2}\left(\left\|u_{n}-v\right\|^{2}+\left\|x_{n}-v\right\|^{2}-\left\|u_{n}-x_{n}\right\|^{2}\right) .
\end{aligned}
$$

It implies that

$$
\left\|u_{n}-v\right\|^{2} \leq\left\|x_{n}-v\right\|^{2}-\left\|u_{n}-x_{n}\right\|^{2}
$$

Since $S_{s_{n}}$ is a firmly nonexpansive mapping and $v_{n}=S_{s_{n}} x_{n}$, by using the same method as (3.34), we have

$$
\left\|v_{n}-v\right\|^{2} \leq\left\|x_{n}-v\right\|^{2}-\left\|v_{n}-x_{n}\right\|^{2} .
$$

Substituting (3.34), (3.35) into (3.33), we have

$$
\begin{aligned}
\left\|x_{n+1}-v\right\|^{2} \leq & \beta_{n}\left\|x_{n}-v\right\|^{2}+\left(1-\beta_{n}\right)\left(\alpha_{n}\left\|f\left(T_{n} y_{n}\right)-v\right\|^{2}\right. \\
& \left.+\left(1-\alpha_{n}\right)\left(\delta_{n}\left\|u_{n}-v\right\|^{2}+\left(1-\delta_{n}\right)\left\|v_{n}-v\right\|^{2}\right)\right) \\
\leq & \beta_{n}\left\|x_{n}-v\right\|^{2}+\left(1-\beta_{n}\right)\left(\alpha_{n}\left\|f\left(T_{n} y_{n}\right)-v\right\|^{2}\right. \\
& +\left(1-\alpha_{n}\right)\left(\delta_{n}\left(\left\|x_{n}-v\right\|^{2}-\left\|u_{n}-x_{n}\right\|^{2}\right)\right.
\end{aligned}
$$




$$
\begin{aligned}
& \left.\left.+\left(1-\delta_{n}\right)\left(\left\|x_{n}-v\right\|^{2}-\left\|v_{n}-x_{n}\right\|^{2}\right)\right)\right) \\
= & \beta_{n}\left\|x_{n}-v\right\|^{2}+\left(1-\beta_{n}\right)\left(\alpha_{n}\left\|f\left(T_{n} y_{n}\right)-v\right\|^{2}\right. \\
& +\left(1-\alpha_{n}\right)\left(\delta_{n}\left\|x_{n}-v\right\|^{2}-\delta_{n}\left\|u_{n}-x_{n}\right\|^{2}\right. \\
& \left.\left.+\left(1-\delta_{n}\right)\left\|x_{n}-v\right\|^{2}-\left(1-\delta_{n}\right)\left\|v_{n}-x_{n}\right\|^{2}\right)\right) \\
= & \beta_{n}\left\|x_{n}-v\right\|^{2}+\left(1-\beta_{n}\right)\left(\alpha_{n}\left\|f\left(T_{n} y_{n}\right)-v\right\|^{2}\right. \\
& +\left(1-\alpha_{n}\right)\left(\left\|x_{n}-v\right\|^{2}-\delta_{n}\left\|u_{n}-x_{n}\right\|^{2}\right. \\
& \left.\left.-\left(1-\delta_{n}\right)\left\|v_{n}-x_{n}\right\|^{2}\right)\right) \\
= & \beta_{n}\left\|x_{n}-v\right\|^{2}+\left(1-\beta_{n}\right) \alpha_{n}\left\|f\left(T_{n} y_{n}\right)-v\right\|^{2} \\
& +\left(1-\alpha_{n}\right)\left(1-\beta_{n}\right)\left(\left\|x_{n}-v\right\|^{2}-\delta_{n}\left\|u_{n}-x_{n}\right\|^{2}\right. \\
& \left.-\left(1-\delta_{n}\right)\left\|v_{n}-x_{n}\right\|^{2}\right) \\
= & \beta_{n}\left\|x_{n}-v\right\|^{2}+\left(1-\beta_{n}\right) \alpha_{n}\left\|f\left(T_{n} y_{n}\right)-v\right\|^{2} \\
& +\left(1-\alpha_{n}\right)\left(1-\beta_{n}\right)\left\|x_{n}-v\right\|^{2}-\delta_{n}\left(1-\alpha_{n}\right)\left(1-\beta_{n}\right)\left\|u_{n}-x_{n}\right\|^{2} \\
& -\left(1-\delta_{n}\right)\left(1-\alpha_{n}\right)\left(1-\beta_{n}\right)\left\|v_{n}-x_{n}\right\|^{2} \\
\leq & \left\|x_{n}-v\right\|^{2}+\alpha_{n}\left\|f\left(T_{n} y_{n}\right)-v\right\|^{2}-\delta_{n}\left(1-\alpha_{n}\right)\left(1-\beta_{n}\right)\left\|u_{n}-x_{n}\right\|^{2} \\
& -\left(1-\delta_{n}\right)\left(1-\alpha_{n}\right)\left(1-\beta_{n}\right)\left\|v_{n}-x_{n}\right\|^{2} .
\end{aligned}
$$

From (3.36), we have

$$
\begin{aligned}
\delta_{n}\left(1-\alpha_{n}\right)\left(1-\beta_{n}\right)\left\|u_{n}-x_{n}\right\|^{2} \leq & \left\|x_{n}-v\right\|^{2}-\left\|x_{n+1}-v\right\|^{2}+\alpha_{n}\left\|f\left(T_{n} y_{n}\right)-v\right\|^{2} \\
& -\left(1-\delta_{n}\right)\left(1-\alpha_{n}\right)\left(1-\beta_{n}\right)\left\|v_{n}-x_{n}\right\|^{2} \\
\leq & \left(\left\|x_{n}-v\right\|+\left\|x_{n+1}-v\right\|\right)\left\|x_{n+1}-x_{n}\right\|+\alpha_{n}\left\|f\left(T_{n} y_{n}\right)-v\right\|^{2} \\
& -\left(1-\delta_{n}\right)\left(1-\alpha_{n}\right)\left(1-\beta_{n}\right)\left\|v_{n}-x_{n}\right\|^{2} \\
\leq & \left(\left\|x_{n}-v\right\|+\left\|x_{n+1}-v\right\|\right)\left\|x_{n+1}-x_{n}\right\| \\
& +\alpha_{n}\left\|f\left(T_{n} y_{n}\right)-v\right\|^{2} .
\end{aligned}
$$

From the conditions (i), (ii), (iv) and (3.17), we have

$$
\lim _{n \rightarrow \infty}\left\|u_{n}-x_{n}\right\|=0 .
$$

By using the method as (3.37), we have

$$
\lim _{n \rightarrow \infty}\left\|v_{n}-x_{n}\right\|=0
$$

From (3.32), (3.37) and (3.38), we have

$$
\lim _{n \rightarrow \infty}\left\|y_{n}-x_{n}\right\|=0 \text {. }
$$

Next, we show that

$$
\left\{y_{n}\right\} \in \widetilde{F}\left(\left\{S_{n}\right\}\right) \cap \widetilde{F}\left(\left\{T_{n}\right\}\right) .
$$


Since

$$
\begin{aligned}
\left\|S_{n} A_{n} T_{n} y_{n}-y_{n}\right\| & \leq\left\|S_{n} A_{n} T_{n} y_{n}-x_{n}\right\|+\left\|x_{n}-y_{n}\right\| \\
& =\left\|w_{n}-x_{n}\right\|+\left\|x_{n}-y_{n}\right\|,
\end{aligned}
$$

from (3.30) and (3.39), we have

$$
\lim _{n \rightarrow \infty}\left\|S_{n} A_{n} T_{n} y_{n}-y_{n}\right\|=0 .
$$

Since $\left\{y_{n}\right\}$ is bounded, we have

$$
\left\{y_{n}\right\} \in \widetilde{F}\left(\left\{S_{n} A_{n} T_{n}\right\}\right) .
$$

Since $\widetilde{F}\left(\left\{S_{n} A_{n} T_{n}\right\}\right)=\widetilde{F}\left(\left\{S_{n}\right\}\right) \cap \widetilde{F}\left(\left\{T_{n}\right\}\right)$ and (3.41), we have (3.40).

Next, we show that

$$
\lim _{n \rightarrow \infty}\left\|S_{n} m_{n}-m_{n}\right\|=0
$$

where $m_{n}=\alpha_{n} f\left(T_{n} y_{n}\right)+\left(1-\alpha_{n}\right) T_{n} y_{n}$. From the definition of $m_{n}$, we have

$$
\begin{aligned}
\left\|S_{n} m_{n}-m_{n}\right\| \leq & \left\|S_{n} m_{n}-x_{n}\right\|+\left\|m_{n}-x_{n}\right\| \\
= & \left\|S_{n} m_{n}-x_{n}\right\|+\left\|\alpha_{n}\left(f\left(T_{n} y_{n}\right)-x_{n}\right)+\left(1-\alpha_{n}\right)\left(T_{n} y_{n}-x_{n}\right)\right\| \\
\leq & \left\|w_{n}-x_{n}\right\|+\alpha_{n}\left\|f\left(T_{n} y_{n}\right)-x_{n}\right\|+\left(1-\alpha_{n}\right)\left\|T_{n} y_{n}-x_{n}\right\| \\
\leq & \left\|w_{n}-x_{n}\right\|+\alpha_{n}\left\|f\left(T_{n} y_{n}\right)-x_{n}\right\| \\
& +\left\|T_{n} y_{n}-y_{n}\right\|+\left\|y_{n}-x_{n}\right\| .
\end{aligned}
$$

From (3.39), (3.40), (3.30) and the condition (i), we have

$$
\lim _{n \rightarrow \infty}\left\|S_{n} m_{n}-m_{n}\right\|=0 .
$$

Next, we show that

$$
\limsup _{n \rightarrow \infty}\left\langle f(z)-z, m_{n}-z\right\rangle \leq 0,
$$

where $z=P_{\mathbb{F}} f(z)$. Since $\left\{y_{n}\right\}$ is bounded, there exists a subsequence $\left\{y_{n_{i}}\right\}$ of $\left\{y_{n}\right\}$ converging weakly to $v$, that is, $y_{n_{i}} \rightarrow v$ as $i \rightarrow \infty$. From (3.40), $\left\{S_{n}\right\}$ and $\left\{T_{n}\right\}$ satisfying the condition (Z), we have $v \in F\left(\left\{S_{n}\right\}\right) \cap F\left(\left\{T_{n}\right\}\right)$.

Define the mapping $Q: C \rightarrow C$ by

$$
Q(x)=\delta S_{r_{n}} x+(1-\delta) S_{S_{n}} x \quad \text { for all } x \in C,
$$

where $\lim _{n \rightarrow \infty} \delta_{n}=\delta \in(0,1)$. From the nonexpansiveness of $S_{r_{n}}, S_{S_{n}}$ and Lemma 2.4, we have

$$
F(Q)=F\left(S_{r_{n}}\right) \cap F\left(S_{S_{n}}\right)=E P\left(F_{1}\right) \cap E P\left(F_{2}\right) .
$$


From the definitions of $y_{n}$ and $Q$, we have

$$
\begin{aligned}
\left\|x_{n}-Q x_{n}\right\| & \leq\left\|x_{n}-y_{n}\right\|+\left\|y_{n}-Q x_{n}\right\| \\
& \leq\left\|x_{n}-y_{n}\right\|+\left\|\delta_{n} u_{n}+\left(1-\delta_{n}\right) v_{n}-\delta S_{r_{n}} x_{n}-(1-\delta) S_{S_{n}} x_{n}\right\| \\
& \leq\left\|x_{n}-y_{n}\right\|+\left|\delta_{n}-\delta\right|\left\|u_{n}\right\|+\left|\delta_{n}-\delta\right|\left\|v_{n}\right\| .
\end{aligned}
$$

From (3.39), (3.42) and the condition (iv), we have

$$
\lim _{n \rightarrow \infty}\left\|x_{n}-Q x_{n}\right\|=0
$$

From (3.39) and $y_{n_{i}} \rightarrow v$ as $i \rightarrow \infty$, we have $x_{n_{i}} \rightarrow v$ as $i \rightarrow \infty$. By (3.43), $x_{n_{i}} \rightarrow v$ as $i \rightarrow \infty$ and Lemma 2.6, we have

$$
v \in F(Q)=E P\left(F_{1}\right) \cap E P\left(F_{2}\right) .
$$

Hence,

$$
v \in E P\left(F_{1}\right) \cap E P\left(F_{2}\right) \cap F\left(\left\{S_{n}\right\}\right) \cap F\left(\left\{T_{n}\right\}\right)=\mathbb{F} .
$$

By (3.40), (3.44) and the condition (i), we have

$$
\begin{aligned}
\limsup _{n \rightarrow \infty}\left\langle f(z)-z, m_{n}-z\right\rangle= & \limsup _{n \rightarrow \infty}\left(\alpha_{n}\left\langle f(z)-z, f\left(T_{n} y_{n}\right)-T_{n} y_{n}\right\rangle\right. \\
& \left.+\left\langle f(z)-z, T_{n} y_{n}-z\right\rangle\right) \\
= & \lim _{i \rightarrow \infty}\left(\alpha_{n_{i}}\left|f(z)-z, f\left(T_{n_{i}} y_{n_{i}}\right)-T_{n_{i}} y_{n_{i}}\right\rangle\right. \\
& \left.+\left\langle f(z)-z, T_{n_{i}} y_{n_{i}}-z\right\rangle\right) \\
= & \lim _{i \rightarrow \infty}\left(\alpha_{n_{i}}\left|f(z)-z, f\left(T_{n_{i}} y_{n_{i}}\right)-T_{n_{i}} y_{n_{i}}\right\rangle\right. \\
& \left.+\left\langle f(z)-z, T_{n_{i}} y_{n_{i}}-y_{n_{i}}\right\rangle+\left\langle f(z)-z, y_{n_{i}}-z\right\rangle\right) \\
= & \langle f(z)-z, v-z\rangle \leq 0 .
\end{aligned}
$$

Finally, we show that the sequence $\left\{x_{n}\right\}$ converges strongly to $z=P_{\mathbb{F}} f(z)$. From the definition of $\left\{x_{n}\right\}$, we have

$$
\begin{aligned}
\left\|x_{n+1}-z\right\|^{2} & =\left\|\beta_{n}\left(x_{n}-z\right)+\left(1-\beta_{n}\right)\left(S_{n} m_{n}-z\right)\right\|^{2} \\
& \leq \beta_{n}\left\|x_{n}-z\right\|^{2}+\left(1-\beta_{n}\right)\left\|S_{n} m_{n}-z\right\|^{2} \\
& \leq \beta_{n}\left\|x_{n}-z\right\|^{2}+\left(1-\beta_{n}\right)\left\|m_{n}-z\right\|^{2} .
\end{aligned}
$$

Since $m_{n}=\alpha_{n} f\left(T_{n} y_{n}\right)+\left(1-\alpha_{n}\right) T_{n} y_{n}$, we have

$$
\begin{aligned}
\left\|m_{n}-z\right\|^{2} & =\left\|\alpha_{n}\left(f\left(T_{n} y_{n}\right)-z\right)+\left(1-\alpha_{n}\right)\left(T_{n} y_{n}-z\right)\right\|^{2} \\
& \leq\left(1-\alpha_{n}\right)^{2}\left\|T_{n} y_{n}-z\right\|^{2}+2 \alpha_{n}\left\langle f\left(T_{n} y_{n}\right)-z, m_{n}-z\right\rangle \\
& \leq\left(1-\alpha_{n}\right)\left\|x_{n}-z\right\|^{2}+2 \alpha_{n}\left\langle f\left(T_{n} y_{n}\right)-f(z), m_{n}-z\right\rangle
\end{aligned}
$$




$$
\begin{aligned}
& +2 \alpha_{n}\left\langle f(z)-z, m_{n}-z\right\rangle \\
\leq & \left(1-\alpha_{n}\right)\left\|x_{n}-z\right\|^{2}+2 \alpha_{n} \alpha\left\|x_{n}-z\right\|\left\|m_{n}-z\right\| \\
& +2 \alpha_{n}\left\langle f(z)-z, m_{n}-z\right\rangle \\
\leq & \left(1-\alpha_{n}\right)\left\|x_{n}-z\right\|^{2}+\alpha_{n} \alpha\left(\left\|x_{n}-z\right\|^{2}+\left\|m_{n}-z\right\|^{2}\right) \\
& +2 \alpha_{n}\left\langle f(z)-z, m_{n}-z\right\rangle \\
= & \left(1-\alpha_{n}\right)\left\|x_{n}-z\right\|^{2}+\alpha_{n} \alpha\left\|x_{n}-z\right\|^{2}+\alpha_{n} \alpha\left\|m_{n}-z\right\|^{2} \\
& +2 \alpha_{n}\left\langle f(z)-z, m_{n}-z\right\rangle \\
= & \left(1-\alpha_{n}(1-\alpha)\right)\left\|x_{n}-z\right\|^{2}+\alpha_{n} \alpha\left\|m_{n}-z\right\|^{2} \\
& +2 \alpha_{n}\left\langle f(z)-z, m_{n}-z\right\rangle .
\end{aligned}
$$

This implies that

$$
\begin{aligned}
\left\|m_{n}-z\right\|^{2} & \leq \frac{1-\alpha_{n}(1-\alpha)}{1-\alpha_{n} \alpha}\left\|x_{n}-z\right\|^{2}+\frac{2 \alpha_{n}}{1-\alpha_{n} \alpha}\left\langle f(z)-z, m_{n}-z\right\rangle \\
& =\frac{1-\alpha_{n} \alpha+\alpha_{n} \alpha-\alpha_{n}(1-\alpha)}{1-\alpha_{n} \alpha}\left\|x_{n}-z\right\|^{2}+\frac{2 \alpha_{n}}{1-\alpha_{n} \alpha}\left\langle f(z)-z, m_{n}-z\right\rangle \\
& =\left(1-\frac{\alpha_{n}(1-2 \alpha)}{1-\alpha_{n} \alpha}\right)\left\|x_{n}-z\right\|^{2}+\frac{2 \alpha_{n}}{1-\alpha_{n} \alpha}\left\langle f(z)-z, m_{n}-z\right\rangle .
\end{aligned}
$$

Substituting (3.46) into (3.45), we have

$$
\begin{aligned}
&\left\|x_{n+1}-z\right\|^{2} \leq \beta_{n}\left\|x_{n}-z\right\|^{2}+\left(1-\beta_{n}\right)\left\|m_{n}-z\right\|^{2} \\
& \leq \beta_{n}\left\|x_{n}-z\right\|^{2}+\left(1-\beta_{n}\right)\left(\left(1-\frac{\alpha_{n}(1-2 \alpha)}{1-\alpha_{n} \alpha}\right)\left\|x_{n}-z\right\|^{2}\right. \\
&\left.+\frac{2 \alpha_{n}}{1-\alpha_{n} \alpha}\left\langle f(z)-z, m_{n}-z\right\rangle\right) \\
& \leq \beta_{n}\left\|x_{n}-z\right\|^{2}+\left(1-\beta_{n}\right)\left(1-\frac{\alpha_{n}(1-2 \alpha)}{1-\alpha_{n} \alpha}\right)\left\|x_{n}-z\right\|^{2} \\
&+\frac{2 \alpha_{n}\left(1-\beta_{n}\right)}{1-\alpha_{n} \alpha}\left\langle f(z)-z, m_{n}-z\right\rangle \\
&= \beta_{n}\left\|x_{n}-z\right\|^{2}+\left(\left(1-\beta_{n}\right)-\frac{\alpha_{n}(1-2 \alpha)\left(1-\beta_{n}\right)}{1-\alpha_{n} \alpha}\right)\left\|x_{n}-z\right\|^{2} \\
&+\frac{2 \alpha_{n}\left(1-\beta_{n}\right)}{1-\alpha_{n} \alpha}\left\langle f(z)-z, m_{n}-z\right\rangle \\
&=\left(1-\frac{\alpha_{n}(1-2 \alpha)\left(1-\beta_{n}\right)}{1-\alpha_{n} \alpha}\right)\left\|x_{n}-z\right\|^{2} \\
&+\frac{\alpha_{n}\left(1-\beta_{n}\right)(1-2 \alpha) 2\left\langle f(z)-z, m_{n}-z\right\rangle}{1-\alpha_{n} \alpha} . \\
&(1-2 \alpha)
\end{aligned}
$$

Applying (3.47), the conditions (i), (ii) and Lemma 2.2, we have $\left\{x_{n}\right\}$ converges strongly to $z=P_{\mathbb{F}} f(z)$. From (3.39), (3.37) and (3.38), it is easy to see that $\left\{y_{n}\right\},\left\{u_{n}\right\},\left\{v_{n}\right\}$ converge strongly to $z=P_{\mathbb{F}} f(z)$. This completes the proof. 


\section{Applications}

In this section, we give three examples for a strongly nonexpansive sequence and prove a strong convergence theorem associated to the variational inequality problem.

Before we give three examples, we need the following definition and lemmas.

Definition 4.1 Let $C$ be a nonempty closed convex subset of a real Hilbert space $H$. A mapping $A: C \rightarrow H$ is called an $\alpha$-inverse strongly monotone mapping if there exists an $\alpha>0$ such that

$$
\langle x-y, a x-A y\rangle \geq \alpha\|A x-A y\|^{2}
$$

for all $x, y \in C$.

A mapping $A: C \rightarrow H$ is called $\alpha$-strongly monotone if there exists $\alpha>0$ such that

$$
\langle x-y, a x-A y\rangle \geq \alpha\|x-y\|^{2}
$$

for all $x, y \in C$

A mapping $T: C \rightarrow C$ is called a $\kappa$-strictly pseudo-contractive mapping if there is $\kappa \in$ $[0,1)$ such that

$$
\|T x-T y\|^{2} \leq\|x-y\|^{2}+\kappa\|(I-T) x-(I-T) y\|^{2}
$$

for all $x, y \in C$.

Then (4.1) is equivalent to

$$
\langle x-y,(I-T) x-(I-T) y\rangle \geq \frac{1-\kappa}{2}\|(I-T) x-(I-T) y\|^{2}
$$

for all $x, y \in C$.

The set of solutions of the variational inequality problem of the mapping $A: C \rightarrow H$ is denoted by $\operatorname{VI}(C, A)$, that is,

$$
V I(C, A)=\{x \in C:\langle y-x, A x\rangle \geq 0, \forall y \in C\}
$$

Let $A, B: C \rightarrow H$ be two mappings. In 2013, Kangtunyakarn [15] modified $V I(C, A)$ as follows:

$$
V I(C, a A+(1-a) B)=\{x \in C:\langle y-x,(a A+(1-a) B) x\rangle \geq 0, \forall y \in C \text { and } a \in(0,1)\} .
$$

Remark 4.1 If $T: C \rightarrow C$ is a $\kappa$-strictly pseudo-contractive mapping with $F(T) \neq \emptyset$, then $(I-T)$ is a $\frac{1-\kappa}{2}$-inverse strongly monotone mapping and $F(T)=V I(C, I-T)$.

Lemma 4.2 (See [16]) Let $H$ be a Hilbert space, let $C$ be a nonempty closed convex subset of $H$, and let $A$ be a mapping of $C$ into $H$. Let $u \in C$. Then, for $\lambda>0$,

$$
u=P_{C}(I-\lambda A) u \quad \Leftrightarrow \quad u \in V I(C, A),
$$

where $P_{C}$ is the metric projection of $H$ onto $C$. 
Lemma 4.3 (See [15]) Let $C$ be a nonempty closed convex subset of a real Hilbert space $H$, and let $A, B: C \rightarrow H$ be $\alpha$ and $\beta$-inverse strongly monotone mappings, respectively, with $\alpha, \beta>0$ and $V I(C, A) \cap V I(C, B) \neq \emptyset$. Then

$$
V I(C, a A+(1-a) B)=V I(C, A) \cap V I(C, B), \quad \forall a \in(0,1) .
$$

Furthermore, if $0<\gamma<2 \eta$, where $\eta=\min \{\alpha, \beta\}$, we have $I-\gamma(a A+(1-a) B)$ is a nonexpansive mapping.

Example 4.4 Let $T: C \rightarrow C$ be a $\kappa$-strictly pseudo-contractive mapping with $F(T) \neq \emptyset$. Let $\left\{\lambda_{n}\right\}$ be a sequence of positive real numbers such that

$$
0<\inf _{n \in \mathbb{N}} \lambda_{n} \leq \sup _{n \in \mathbb{N}} \lambda_{n}<1-\kappa \text { and } \lim _{n \rightarrow \infty}\left(\lambda_{n+1}-\lambda_{n}\right)=0,
$$

and let $\left\{T_{n}\right\}$ be a sequence of mappings defined by $T_{n}=P_{C}\left(I-\lambda_{n}(I-T)\right)$. Then $\left\{T_{n}\right\}$ is a strongly nonexpansive sequence satisfying the conditions $(R)$ and $(Z)$.

Proof Since $T$ is a $\kappa$-strictly pseudo-contractive mapping, then $I-T$ is $\frac{1-\kappa}{2}$-inverse strongly monotone. From Example 4.3 in [10], we have $\left\{T_{n}\right\}$ is a strongly nonexpansive sequence satisfying the conditions $(R)$ and $(Z)$.

Example 4.5 Let $A, B: C \rightarrow H$ be $\alpha, \beta$-inverse strongly monotone mappings, respectively, with $\bar{\gamma}=\min \{\alpha, \beta\}$ and $V I(C, A) \cap V I(C, B) \neq \emptyset$. Let $\left\{\lambda_{n}\right\}$ be a sequence of positive real numbers such that

$$
0<\inf _{n \in \mathbb{N}} \lambda_{n} \leq \sup _{n \in \mathbb{N}} \lambda_{n}<2 \bar{\gamma} \quad \text { and } \quad \lim _{n \rightarrow \infty}\left(\lambda_{n+1}-\lambda_{n}\right)=0,
$$

and let $\left\{T_{n}\right\}$ be a sequence of mappings defined by $T_{n}=P_{C}\left(I-\lambda_{n} D\right)$, where $D=a A+(1-a) B$ for all $a \in(0,1)$. Then $\left\{T_{n}\right\}$ is a strongly nonexpansive sequence satisfying the conditions $(R)$ and $(Z)$.

Proof Let $x, y \in C$, then we have

$$
\begin{aligned}
\langle x-y, D x-D y\rangle & =\langle x-y,(a A+(1-a) B) x-(a A+(1-a) B) y\rangle \\
& \geq a\langle x-y, A x-A y\rangle+(1-a)\langle x-y, B x-B y\rangle \\
& \geq a \alpha\|A x-A y\|^{2}+(1-a) \beta\|B x-B y\|^{2} \\
& \geq \bar{\gamma}\left(\|a A x+(1-a) B x-a A y-(1-a) B y\|^{2}\right) \\
& \geq \bar{\gamma}\|D x-D y\|^{2} .
\end{aligned}
$$

Then $D$ is a $\bar{\gamma}$-inverse strongly monotone mapping. From Example 4.3 in [10], we have that $\left\{T_{n}\right\}$ is a strongly nonexpansive sequence satisfying the conditions $(R)$ and $(Z)$.

Example 4.6 Let $A: C \rightarrow H$ be an $\alpha$-strongly monotone and $L$-Lipschitzian mapping with $V I(C, A) \neq \emptyset$. Let $\left\{\lambda_{n}\right\}$ be a sequence of positive real numbers such that

$$
0<\inf _{n \in \mathbb{N}} \lambda_{n} \leq \sup _{n \in \mathbb{N}} \lambda_{n}<\frac{2 \alpha}{L^{2}} \quad \text { and } \quad \lim _{n \rightarrow \infty}\left(\lambda_{n+1}-\lambda_{n}\right)=0,
$$


and let $\left\{T_{n}\right\}$ be a sequence of mappings defined by $T_{n}=P_{C}\left(I-\lambda_{n} A\right)$. Then $\left\{T_{n}\right\}$ is a strongly nonexpansive sequence satisfying the conditions $(R)$ and $(Z)$.

Proof Let $x, y \in C$, then we have

$$
\begin{aligned}
\langle x-y, A x-A y\rangle & \geq \alpha\|x-y\|^{2} \\
& \geq \frac{\alpha}{L^{2}}\|A x-A y\|^{2} .
\end{aligned}
$$

Then $A$ is an $\frac{\alpha}{L^{2}}$-inverse strongly monotone mapping. From Example 4.3 in [10], we have that $\left\{T_{n}\right\}$ is a strongly nonexpansive sequence satisfying the conditions $(R)$ and $(Z)$.

Example 4.7 (See [10]) Let $\left\{R_{n}\right\}$ be a sequence of nonexpansive mappings of $C$ into itself having a common fixed point, and let $\left\{\mu_{n}\right\}$ be a sequence in $[0,1]$. For each $n \in \mathbb{N}$, a $W$ mapping [17] $T_{n}$ generated by $R_{n}, R_{n-1}, \ldots, R_{1}$ and $\mu_{n}, \mu_{n-1}, \ldots, \mu_{1}$ is defined as follows:

$$
\begin{aligned}
& U_{n, n}=\mu_{n} R_{n}+\left(1-\mu_{n}\right) I, \\
& U_{n, n-1}=\mu_{n-1} R_{n-1} U_{n, n}+\left(1-\mu_{n-1}\right) I, \\
& U_{n, n-2}=\mu_{n-2} R_{n-2} U_{n, n-1}+\left(1-\mu_{n-2}\right) I, \\
& \vdots \\
& U_{n, k}=\mu_{k} R_{k} U_{n, k+1}+\left(1-\mu_{k}\right) I, \\
& \vdots \\
& U_{n, 2}=\mu_{2} R_{2} U_{n, 3}+\left(1-\mu_{2}\right) I, \\
& T_{n}=U_{n, 1}=\mu_{1} R_{1} U_{n, 2}+\left(1-\mu_{1}\right) I .
\end{aligned}
$$

If $0<\mu_{1} \leq 1$ and $0<\mu_{n} \leq b$, for all $n \geq 2$ and $0<b<1$, then $\left\{T_{n}\right\}$ satisfies the conditions $(R)$ and $(Z)$.

By using our main result and these three examples, we obtain the following results.

Theorem 4.8 Let $H$ be a Hilbert space, let $C$ be a nonempty closed convex subset of $H$. Let $F_{1}$ and $F_{2}$ be two bifunctions from $C \times C$ into $\mathbb{R}$ satisfying (A1)-(A4), respectively. Let $T: C \rightarrow C$ be a $\kappa$-strictly pseudo-contractive mapping with $F(T) \neq \emptyset$. Let $\left\{\lambda_{n}\right\}$ be a sequence of positive real numbers such that

$$
0<\inf _{n \in \mathbb{N}} \lambda_{n} \leq \sup _{n \in \mathbb{N}} \lambda_{n}<1-\kappa \quad \text { and } \quad \lim _{n \rightarrow \infty}\left(\lambda_{n+1}-\lambda_{n}\right)=0 \text {, }
$$

and let $\left\{T_{n}\right\}$ be a sequence of mappings defined by $T_{n}=P_{C}\left(I-\lambda_{n}(I-T)\right)$. Let $\left\{R_{n}\right\}$ be a sequence of nonexpansive mappings of $C$ into itself having a common fixed point, and let $\left\{\mu_{n}\right\}$ be a sequence in $[0,1]$. For each $n \in \mathbb{N}, W_{n}$ is a $W$-mapping generated by $R_{n}, R_{n-1}, \ldots, R_{1}$ and $\mu_{n}, \mu_{n-1}, \ldots, \mu_{1}$. Assume that $\mathbb{F}=E P\left(F_{1}\right) \cap E P\left(F_{2}\right) \cap F\left(\left\{R_{n}\right\}\right) \cap F(T) \neq \emptyset$. Let $f: C \rightarrow C$ be a contractive mapping with $\alpha \in\left(0, \frac{1}{2}\right)$. Let $\left\{x_{n}\right\},\left\{u_{n}\right\},\left\{v_{n}\right\}$ be sequences generated by 
$x_{1}, u, v \in C$ and

$$
\left\{\begin{array}{l}
F_{1}\left(u_{n}, u\right)+\frac{1}{r_{n}}\left\langle u-u_{n}, u_{n}-x_{n}\right\rangle \geq 0, \\
F_{2}\left(v_{n}, v\right)+\frac{1}{s_{n}}\left\langle v-v_{n}, v_{n}-x_{n}\right\rangle \geq 0, \\
y_{n}=\delta_{n} u_{n}+\left(1-\delta_{n}\right) v_{n}, \\
x_{n+1}=\beta_{n} x_{n}+\left(1-\beta_{n}\right) W_{n}\left(\alpha_{n} f\left(T_{n} y_{n}\right)+\left(1-\alpha_{n}\right) T_{n} y_{n}\right), \quad \forall n \geq 1,
\end{array}\right.
$$

where $\left\{\alpha_{n}\right\},\left\{\beta_{n}\right\} \in[0,1],\left\{r_{n}\right\},\left\{s_{n}\right\} \in(a, b) \in[0,1]$. Assume that the following conditions hold:

(i) $\lim _{n \rightarrow \infty} \alpha_{n}=0$ and $\sum_{n=1}^{\infty} \alpha_{n}=\infty$;

(ii) $0<\liminf _{n \rightarrow \infty} \beta_{n} \leq \limsup _{n \rightarrow \infty} \beta_{n}<1$;

(iii) $\sum_{n=0}^{\infty}\left|r_{n+1}-r_{n}\right|, \sum_{n=0}^{\infty}\left|s_{n+1}-s_{n}\right|<\infty$;

(iv) $\lim _{n \rightarrow \infty} \delta_{n}=\delta \in(0,1)$.

Then the sequences $\left\{x_{n}\right\},\left\{u_{n}\right\},\left\{v_{n}\right\},\left\{y_{n}\right\}$ converge strongly to $z=P_{\mathbb{F}} f(z)$.

Proof From Example 4.4, we have $\left\{T_{n}\right\}$ is a strongly nonexpansive sequence satisfying the conditions $(R)$ and $(Z)$. From Lemma 4.2, we have $F\left(T_{n}\right)=F\left(P_{C}\left(I-\lambda_{n}(I-T)\right)\right)=$ $V I(C, I-T)=F(T)$ for all $n \in \mathbb{N}$. It implies that $F\left(\left\{T_{n}\right\}\right)=F(T)$. From [18], we have $F\left(\left\{W_{n}\right\}\right)=F\left(\left\{R_{n}\right\}\right)$. It follows that $\mathbb{F}=E P\left(F_{1}\right) \cap E P\left(F_{2}\right) \cap F\left(\left\{W_{n}\right\}\right) \cap F\left(\left\{T_{n}\right\}\right) \neq \emptyset$. From Example 4.7, we have $\left\{W_{n}\right\}$ is a nonexpansive sequence satisfying the conditions $(R)$ and $(Z)$. By Theorem 3.1, we can conclude the desired result.

Theorem 4.9 Let $H$ be a Hilbert space, let $C$ be a nonempty closed convex subset of $H$. Let $F_{1}$ and $F_{2}$ be two bifunctions from $C \times C$ into $\mathbb{R}$ satisfying (A1)-(A4), respectively. Let $A, B: C \rightarrow H$ be $\alpha, \beta$-inverse strongly monotone mappings, respectively, with $\bar{\gamma}=\min \{\alpha, \beta\}$ and $\operatorname{VI}(C, A) \cap \operatorname{VI}(C, B) \neq \emptyset$. Let $\left\{\lambda_{n}\right\}$ be a sequence of positive real numbers such that

$$
0<\inf _{n \in \mathbb{N}} \lambda_{n} \leq \sup _{n \in \mathbb{N}} \lambda_{n}<2 \bar{\gamma} \quad \text { and } \quad \lim _{n \rightarrow \infty}\left(\lambda_{n+1}-\lambda_{n}\right)=0
$$

and let $\left\{T_{n}\right\}$ be a sequence of mappings defined by $T_{n}=P_{C}\left(I-\lambda_{n} D\right)$, where $D=a A+(1-a) B$ for all $a \in(0,1)$. Let $\left\{R_{n}\right\}$ be a sequence of nonexpansive mappings of $C$ into itself having a common fixed point, and let $\left\{\mu_{n}\right\}$ be a sequence in $[0,1]$. For each $n \in \mathbb{N}, W_{n}$ is a $W$-mapping generated by $R_{n}, R_{n-1}, \ldots, R_{1}$ and $\mu_{n}, \mu_{n-1}, \ldots, \mu_{1}$. Assume that $\mathbb{F}=E P\left(F_{1}\right) \cap$ $E P\left(F_{2}\right) \cap F\left(\left\{R_{n}\right\}\right) \cap V I(C, A) \cap V I(C, B) \neq \emptyset$. Let $f: C \rightarrow C$ be a contractive mapping with $\alpha \in\left(0, \frac{1}{2}\right)$. Let $\left\{x_{n}\right\},\left\{u_{n}\right\},\left\{v_{n}\right\}$ be sequences generated by $x_{1}, u, v \in C$ and

$$
\left\{\begin{array}{l}
F_{1}\left(u_{n}, u\right)+\frac{1}{r_{n}}\left\langle u-u_{n}, u_{n}-x_{n}\right\rangle \geq 0, \\
F_{2}\left(v_{n}, v\right)+\frac{1}{s_{n}}\left\langle v-v_{n}, v_{n}-x_{n}\right\rangle \geq 0, \\
y_{n}=\delta_{n} u_{n}+\left(1-\delta_{n}\right) v_{n}, \\
x_{n+1}=\beta_{n} x_{n}+\left(1-\beta_{n}\right) W_{n}\left(\alpha_{n} f\left(T_{n} y_{n}\right)+\left(1-\alpha_{n}\right) T_{n} y_{n}\right), \quad \forall n \geq 1,
\end{array}\right.
$$

where $\left\{\alpha_{n}\right\},\left\{\beta_{n}\right\} \in[0,1],\left\{r_{n}\right\},\left\{s_{n}\right\} \in(a, b) \in[0,1]$. Assume that the following conditions hold:

(i) $\lim _{n \rightarrow \infty} \alpha_{n}=0$ and $\sum_{n=1}^{\infty} \alpha_{n}=\infty$;

(ii) $0<\liminf _{n \rightarrow \infty} \beta_{n} \leq \operatorname{lim\operatorname {sup}_{n\rightarrow \infty }} \beta_{n}<1$; 
(iii) $\sum_{n=0}^{\infty}\left|r_{n+1}-r_{n}\right|, \sum_{n=0}^{\infty}\left|s_{n+1}-s_{n}\right|<\infty$;

(iv) $\lim _{n \rightarrow \infty} \delta_{n}=\delta \in(0,1)$.

Then the sequences $\left\{x_{n}\right\},\left\{u_{n}\right\},\left\{v_{n}\right\},\left\{y_{n}\right\}$ converge strongly to $z=P_{\mathbb{F}} f(z)$.

Proof From Example 4.5, we have $\left\{T_{n}\right\}$ is a strongly nonexpansive sequence satisfying the conditions $(R)$ and $(Z)$. From Lemmas 4.2 and 4.3, we have $F\left(T_{n}\right)=F\left(P_{C}\left(I-\lambda_{n} D\right)\right)=$ $V I(C, D)=V I(C, A) \cap V I(C, B)$ for all $n \in \mathbb{N}$. It implies that $F\left(\left\{T_{n}\right\}\right)=V I(C, A) \cap V I(C, B)$. From [18], we have $F\left(\left\{W_{n}\right\}\right)=F\left(\left\{R_{n}\right\}\right)$. It follows that $\mathbb{F}=E P\left(F_{1}\right) \cap E P\left(F_{2}\right) \cap F\left(\left\{W_{n}\right\}\right) \cap$ $F\left(\left\{T_{n}\right\}\right) \neq \emptyset$. From Example 4.7, we have $\left\{W_{n}\right\}$ is a nonexpansive sequence satisfying the conditions $(R)$ and $(Z)$. By Theorem 3.1, we can conclude the desired result.

Theorem 4.10 Let $H$ be a Hilbert space, let $C$ be a nonempty closed convex subset of $H$. Let $F_{1}$ and $F_{2}$ be two bifunctions from $C \times C$ into $\mathbb{R}$ satisfying (A1)-(A4), respectively. Let $A: C \rightarrow H$ be an $\alpha$-strongly monotone and L-Lipschitzian mapping with $V I(C, A) \neq \emptyset$. Let $\left\{\lambda_{n}\right\}$ be a sequence of positive real numbers such that

$$
0<\inf _{n \in \mathbb{N}} \lambda_{n} \leq \sup _{n \in \mathbb{N}} \lambda_{n}<\frac{2 \alpha}{L^{2}} \quad \text { and } \quad \lim _{n \rightarrow \infty}\left(\lambda_{n+1}-\lambda_{n}\right)=0
$$

and let $\left\{T_{n}\right\}$ be a sequence of mappings defined by $T_{n}=P_{C}\left(I-\lambda_{n} A\right)$. Let $\left\{R_{n}\right\}$ be a sequence of nonexpansive mappings of $C$ into itself having a common fixed point, and let $\left\{\mu_{n}\right\}$ be a sequence in $[0,1]$. For each $n \in \mathbb{N}, W_{n}$ is a $W$-mapping generated by $R_{n}, R_{n-1}, \ldots, R_{1}$ and $\mu_{n}, \mu_{n-1}, \ldots, \mu_{1}$. Assume that $\mathbb{F}=E P\left(F_{1}\right) \cap E P\left(F_{2}\right) \cap F\left(\left\{R_{n}\right\}\right) \cap V I(C, A) \neq \emptyset$. Let $f: C \rightarrow C$ be a contractive mapping with $\alpha \in\left(0, \frac{1}{2}\right)$. Let $\left\{x_{n}\right\},\left\{u_{n}\right\},\left\{v_{n}\right\}$ be sequences generated by $x_{1}, u, v \in C$ and

$$
\left\{\begin{array}{l}
F_{1}\left(u_{n}, u\right)+\frac{1}{r_{n}}\left\langle u-u_{n}, u_{n}-x_{n}\right\rangle \geq 0, \\
F_{2}\left(v_{n}, v\right)+\frac{1}{s_{n}}\left\langle v-v_{n}, v_{n}-x_{n}\right\rangle \geq 0, \\
y_{n}=\delta_{n} u_{n}+\left(1-\delta_{n}\right) v_{n}, \\
x_{n+1}=\beta_{n} x_{n}+\left(1-\beta_{n}\right) W_{n}\left(\alpha_{n} f\left(T_{n} y_{n}\right)+\left(1-\alpha_{n}\right) T_{n} y_{n}\right), \quad \forall n \geq 1,
\end{array}\right.
$$

where $\left\{\alpha_{n}\right\},\left\{\beta_{n}\right\} \in[0,1],\left\{r_{n}\right\},\left\{s_{n}\right\} \in(a, b) \in[0,1]$. Assume that the following conditions hold:

(i) $\lim _{n \rightarrow \infty} \alpha_{n}=0$ and $\sum_{n=1}^{\infty} \alpha_{n}=\infty$;

(ii) $0<\liminf _{n \rightarrow \infty} \beta_{n} \leq \limsup _{n \rightarrow \infty} \beta_{n}<1$;

(iii) $\sum_{n=0}^{\infty}\left|r_{n+1}-r_{n}\right|, \sum_{n=0}^{\infty}\left|s_{n+1}-s_{n}\right|<\infty$;

(iv) $\lim _{n \rightarrow \infty} \delta_{n}=\delta \in(0,1)$.

Then the sequences $\left\{x_{n}\right\},\left\{u_{n}\right\},\left\{v_{n}\right\},\left\{y_{n}\right\}$ converge strongly to $z=P_{\mathbb{F}} f(z)$.

Proof From Example 4.6, we have $\left\{T_{n}\right\}$ is a strongly nonexpansive sequence satisfying the conditions $(R)$ and $(Z)$. From Lemma 4.2, we have $F\left(T_{n}\right)=F\left(P_{C}\left(I-\lambda_{n} A\right)\right)=V I(C, A)$ for all $n \in \mathbb{N}$. It implies that $F\left(\left\{T_{n}\right\}\right)=V I(C, A)$. From [18], we have $F\left(\left\{W_{n}\right\}\right)=F\left(\left\{R_{n}\right\}\right)$. It follows that $\mathbb{F}=E P\left(F_{1}\right) \cap E P\left(F_{2}\right) \cap F\left(\left\{W_{n}\right\}\right) \cap F\left(\left\{T_{n}\right\}\right) \neq \emptyset$. From Example 4.7, we have $\left\{W_{n}\right\}$ is a nonexpansive sequence satisfying the conditions $(R)$ and $(Z)$. By Theorem 3.1, we can conclude the desired result. 
Theorem 4.11 Let $H$ be a Hilbert space, let $C$ be a nonempty closed convex subset of H. Let $F_{1}$ be a bifunction from $C \times C$ into $\mathbb{R}$ satisfying (A1)-(A4), and let $\left\{S_{n}\right\}$ and $\left\{T_{n}\right\}$ be sequences of nonexpansive self-mappings of $C$ with $\mathbb{F}=E P\left(F_{1}\right) \cap F\left(\left\{S_{n}\right\}\right) \cap F\left(\left\{T_{n}\right\}\right) \neq \emptyset$. Let $\left\{T_{n}\right\}$ or $\left\{S_{n}\right\}$ be a sequence of strongly nonexpansive mappings, and let $f: C \rightarrow C$ be a contractive mapping with $\alpha \in\left(0, \frac{1}{2}\right)$. Let $\left\{x_{n}\right\},\left\{u_{n}\right\}$ be sequences generated by $x_{1}, u \in C$ and

$$
\left\{\begin{array}{l}
F_{1}\left(u_{n}, u\right)+\frac{1}{r_{n}}\left\langle u-u_{n}, u_{n}-x_{n}\right\rangle \geq 0, \\
x_{n+1}=\beta_{n} x_{n}+\left(1-\beta_{n}\right) S_{n}\left(\alpha_{n} f\left(T_{n} u_{n}\right)+\left(1-\alpha_{n}\right) T_{n} u_{n}\right), \quad \forall n \geq 1,
\end{array}\right.
$$

where $\left\{\alpha_{n}\right\},\left\{\beta_{n}\right\} \in[0,1],\left\{r_{n}\right\},\left\{s_{n}\right\} \in(a, b) \in[0,1]$. Assume that the following conditions hold:

(i) $\lim _{n \rightarrow \infty} \alpha_{n}=0$ and $\sum_{n=1}^{\infty} \alpha_{n}=\infty$;

(ii) $0<\liminf _{n \rightarrow \infty} \beta_{n} \leq \lim \sup _{n \rightarrow \infty} \beta_{n}<1$;

(iii) $\sum_{n=0}^{\infty}\left|r_{n+1}-r_{n}\right|<\infty$;

(iv) $\left\{S_{n}\right\}$ and $\left\{T_{n}\right\}$ satisfy the conditions $R$ and $Z$.

Then the sequences $\left\{x_{n}\right\},\left\{u_{n}\right\}$ converge strongly to $z=P_{\mathbb{F}} f(z)$.

Proof Put $F_{1} \equiv F_{2}, s_{n}=r_{n}$ and $u_{n}=v_{n}$. From Theorem 3.1, we can conclude the desired conclusion.

The following result can be obtained from Theorem 3.1. We, therefore, omit the proof.

Theorem 4.12 Let $H$ be a Hilbert space, let $C$ be a nonempty closed convex subset of $H$. Let $F_{i}$ be bifunctions from $C \times C$ into $\mathbb{R}$, for every $i=1,2, \ldots, N$, satisfying (A1)-(A4), and let $\left\{S_{n}\right\}$ and $\left\{T_{n}\right\}$ be sequences of nonexpansive self-mappings of $C$ with $\mathbb{F}=\bigcap_{i=1}^{N} E P\left(F_{i}\right) \cap$ $F\left(\left\{S_{n}\right\}\right) \cap F\left(\left\{T_{n}\right\}\right) \neq \emptyset$. Let $\left\{T_{n}\right\}$ or $\left\{S_{n}\right\}$ be a sequence of strongly nonexpansive mappings, and let $f: C \rightarrow C$ be a contractive mapping with $\alpha \in\left(0, \frac{1}{2}\right)$. Let $\left\{x_{n}\right\},\left\{u_{n}\right\},\left\{v_{n}\right\}$ be sequences generated by $x_{1}, u^{i} \in C$, for every $i \in 1,2, \ldots, N$, and

$$
\left\{\begin{array}{l}
F_{i}\left(u_{n}^{i}, u^{i}\right)+\frac{1}{r_{n}^{i}}\left\langle u-u_{n}^{i}, u_{n}^{i}-x_{n}\right\rangle \geq 0, \\
y_{n}=\sum_{i=1}^{N} \delta_{n}^{i} u_{n}^{i}, \\
x_{n+1}=\beta_{n} x_{n}+\left(1-\beta_{n}\right) S_{n}\left(\alpha_{n} f\left(T_{n} y_{n}\right)+\left(1-\alpha_{n}\right) T_{n} y_{n}\right), \quad \forall n \geq 1,
\end{array}\right.
$$

where $\left\{\alpha_{n}\right\},\left\{\beta_{n}\right\} \in[0,1],\left\{r_{n}\right\},\left\{s_{n}\right\} \in(a, b) \in[0,1]$. Assume that the following conditions hold:

(i) $\lim _{n \rightarrow \infty} \alpha_{n}=0$ and $\sum_{n=1}^{\infty} \alpha_{n}=\infty$;

(ii) $0<\liminf _{n \rightarrow \infty} \beta_{n} \leq \limsup _{n \rightarrow \infty} \beta_{n}<1$;

(iii) $\sum_{n=0}^{\infty}\left|r_{n+1}^{i}-r_{n}^{i}\right|<\infty, \forall i=1,2, \ldots, N$;

(iv) $\sum_{i=1}^{N} \delta_{n}^{i}=1$;

(v) $\lim _{n \rightarrow \infty} \delta_{n}^{i}=\delta^{i} \in(0,1), \forall i=1,2, \ldots, N$;

(vi) $\left\{S_{n}\right\}$ and $\left\{T_{n}\right\}$ satisfy the conditions $R$ and $Z$.

Then the sequences $\left\{x_{n}\right\},\left\{y_{n}\right\}$ and $\left\{u_{n}^{i}\right\}$, for every $i=1,2, \ldots, N$, converge strongly to $z=$ $P_{\mathbb{F}} f(z)$. 


\section{Competing interests}

The author declares that they have no competing interests.

\section{Acknowledgements}

This research was supported by the Research Administration Division of King Mongkut's Institute of Technology Ladkrabang.

Received: 29 August 2012 Accepted: 9 July 2013 Published: 22 July 2013

References

1. Aoyama, K, Kimura, Y, Takahashi, W, Toyoda, M: On a strongly nonexpansive sequence in Hilbert spaces. J. Nonlinear Convex Anal. 8, 471-489 (2007)

2. Aoyama, K: An iterative method for fixed point problems for sequences of nonexpansive mappings. In: Fixed Point Theory and Applications, pp. 1-7. Yokohama Publ., Yokohama (2010)

3. Blum, E, Oettli, W: From optimization and variational inequalities to equilibrium problems. Math. Stud. 63(1-4), 123-145 (1994)

4. Combettes, PL, Hirstoaga, SA: Equilibrium programming in Hilbert spaces. J. Nonlinear Convex Anal. 6(1), 117-136 (2005)

5. Kangtunyakarn, A: Iterative methods for finding common solution of generalized equilibrium problems and variational inequality problems and fixed point problems of a finite family of nonexpansive mappings. Fixed Point Theory Appl. 2010, Article ID 836714 (2010). doi:10.1155/2010/836714

6. Kangtunyakarn, A: Hybrid algorithm for finding common elements of the set of generalized equilibrium problems and the set of fixed point problems of strictly pseudocontractive mapping. Fixed Point Theory Appl. 2011, Article ID 274820 (2011). doi:10.1155/2011/274820

7. Cholamjiak, W, Suantai, S: A hybrid method for a countable family of multivalued maps, equilibrium problems, and variational inequality problems. Discrete Dyn. Nat. Soc. 2010, Article ID 349158 (2010). doi:10.1155/2010/349158

8. Takahashi, W, Zembayashi, K: Strong convergence theorem by a new hybrid method for equilibrium problems and relatively nonexpansive mappings. Fixed Point Theory Appl. 2008, Article ID 528476 (2008). doi:10.1155/2008/528476

9. Takahashi, S, Takahashi, W: Viscosity approximation methods for equilibrium problems and fixed point problems in Hilbert spaces. J. Math. Anal. Appl. 331(1), 506-515 (2007)

10. Aoyama, K, Kimura, Y: Strong convergence theorems for strongly nonexpansive sequences. Appl. Math. Comput. 217, 7537-7545 (2011)

11. Browder, FE: Convergence of approximants to fixed points of nonexpansive nonlinear mappings in Banach space. Arch. Ration. Mech. Anal. 24, 82-89 (1967)

12. Xu, HK: An iterative approach to quadratic optimization. J. Optim. Theory Appl. 116(3), 659-678 (2003)

13. Suzuki, T: Strong convergence of Krasnoselskii and Mann's type sequences for one-parameter nonexpansive semigroups without Bochner integrals. J. Math. Anal. Appl. 305(1), 227-239 (2005)

14. Bruck, RE: Properties of fixed point sets of nonexpansive mappings in Banach spaces. Trans. Am. Math. Soc. 179 251-262 (1973)

15. Kangtunyakarn, A: Convergence theorem of $\kappa$-strictly pseudocontractive mapping and a modification of generalized equilibrium problems. Fixed Point Theory Appl. 2012, Article ID 89 (2012)

16. Takahashi, W: Nonlinear Functional Analysis. Yokohama Publ, Yokohama (2000)

17. Takahashi, W: Weak and strong convergence theorems for families of nonexpansive mappings and their applications In: Proceedings of Workshop on Fixed Point Theory Kazimierz Dolny, pp. 277-292 (1997)

18. Atsushiba, S, Takahashi, W: Strong convergence theorems for a finite family of nonexpansive mappings and applications. Indian J. Math. 41, 435-453 (1999)

doi:10.1186/1687-1812-2013-193

Cite this article as: Kangtunyakarn: Convergence theorems for a system of equilibrium problems and fixed point problems of a strongly nonexpansive sequence. Fixed Point Theory and Applications 2013 2013:193.

\section{Submit your manuscript to a SpringerOpen ${ }^{\circ}$ journal and benefit from:}

- Convenient online submission

- Rigorous peer review

- Immediate publication on acceptance

- Open access: articles freely available online

- High visibility within the field

- Retaining the copyright to your article 